Supporting Information

for

\title{
A Random Forest Predictor for Diblock Copolymer Phase Behavior
}

\author{
Akash Arora, Tzyy-Shyang Lin, Nathan J. Rebello, Sarah H. M. Av-Ron, Hidenobu Mochigase, and \\ Bradley D. Olsen* \\ Department of Chemical Engineering, Massachusetts Institute of Technology, \\ 77 Massachusetts Avenue, Cambridge, Massachusetts 02139, United States
}

E-mail: bdolsen@mit.edu

\section{Group Contribution Theory Calculations}

Flory and Huggins defined $\chi_{A B}$ as a purely enthalpic quantity accounting for the excess free energy of mixing of chemically-distinct blocks A and B arising from their segment-segment interactions. The most direct approach to estimate $\chi_{A B}$ is,

$$
\chi_{A B}=\frac{v_{\mathrm{ref}}}{k_{B} T}\left(\delta_{A}-\delta_{B}\right)^{2}
$$

where $\delta_{i}=\sqrt{C E D_{i}}$ is the Hildebrand solubility parameter of block $i$. Several experimental studies observed that the above form cannot explain the phase behavior of many commonly-used block copolymers. Accordingly, a constant representing the entropic contribution to $\chi_{A B}$ is added, ${ }^{1}$ resulting in the following functional form:

$$
\chi_{A B}=\frac{v_{\text {ref }}}{k_{B} T}\left(\delta_{A}-\delta_{B}\right)^{2}+0.34
$$

The constant 0.34 is an empirical estimate which is observed to capture the phase behavior of many common block copolymers and polymer blends. A more general form,

$$
\chi_{A B}=\frac{\alpha}{T}+\beta,
$$

where the constants $\alpha$ and $\beta$ are specific to chemistry pair (A, B) and are obtained by fitting a series of experiments to theoretical predictions. For all the RFC model results presented in this work, Eq. (2) is used to calculate $\chi_{A B}$, with $\delta_{A}$ and $\delta_{B}$ obtained using the group contribution theory (GCT), and the reference volume is assumed as $v_{\text {ref }}=100 \AA^{3}$. Accordingly, this method is referred to as GCT for indicating the type of fingerprint used. Inspired by pioneering work in early 1970 's, ${ }^{2,3}$ the parameters $\delta_{A}$ and $\delta_{B}$ are calculated with the groups and their contributions included in Table S1.

Table S1: Values of solubility parameters calculated using the groups and their contributions proposed by Fedors and reported by van Krevelen $e t a l .^{2}$ The solubility parameter is calculated as, $\delta_{i}=\left(C E D_{i} / v_{i}\right)^{1 / 2}$, where $v_{i}=M_{0, i} / \rho_{i}$ is the volume of polymer's repeat unit with $M_{0, i}$ being the repeat-unit molar mass, and $\rho_{i}$ being the bulk density.

\begin{tabular}{lccccc}
\hline \hline Polymer & Chemical Groups & $\begin{array}{c}\rho \\
\left(\mathrm{g} / \mathrm{cm}^{3}\right)\end{array}$ & $\begin{array}{c}v \\
\left(\mathrm{~cm}^{3} / \mathrm{mol}\right)\end{array}$ & $\begin{array}{c}\text { CED } \\
(\mathrm{J} / \mathrm{mol})\end{array}$ & $\begin{array}{c}\delta \\
\left(\mathrm{J} / \mathrm{cm}^{3}\right)^{1 / 2}\end{array}$ \\
\hline
\end{tabular}




\begin{tabular}{|c|c|c|c|c|c|}
\hline Poly(styrene) & $1 \mathrm{C}_{6} \mathrm{H}_{6}+1(>\mathrm{CH}-)+1\left(-\mathrm{CH}_{2}-\right)$ & 0.909 & 114.40 & 40310 & 18.77 \\
\hline Poly(dimethyl siloxane) & $2 \mathrm{C}_{6} \mathrm{H}_{6}+1 \mathrm{Si}+10$ & 0.965 & 76.68 & 19510 & 15.95 \\
\hline $\begin{array}{l}\text { Poly(ethylene glycol) or } \\
\text { Poly(ethylene oxide) }\end{array}$ & $1 \mathrm{CH}_{2}+10$ & 1.125 & 39.10 & 13230 & 18.40 \\
\hline Poly(ethylene) & $2 \mathrm{CH}_{2}$ & 0.900 & 31.10 & 4940 & 12.60 \\
\hline Poly(ethyl ethylene) & $1 \mathrm{CH}_{3}+2 \mathrm{CH}_{2}+1 \mathrm{CH}$ & 0.950 & 58.95 & 18020 & 17.48 \\
\hline Poly(ethyl propylene) & $1 \mathrm{CH}_{3}+3 \mathrm{CH}_{2}+1 \mathrm{CH}$ & 0.860 & 81.40 & 22960 & 16.79 \\
\hline Poly(lactic acid) & $1 \mathrm{CH}_{2}+1 \mathrm{CH}_{3}+1 \mathrm{COO}$ & 1.210 & 47.11 & 27650 & 24.23 \\
\hline Poly(1,4-butadiene) & $2 \mathrm{CH}_{2}+2(=\mathrm{CH})$ & 0.890 & 58.42 & 18500 & 17.80 \\
\hline Poly(1,2-butadiene) & $1 \mathrm{CH}_{2}+1 \mathrm{CH}+1(=\mathrm{CH})+1\left(=\mathrm{CH}_{2}\right)$ & 0.950 & 54.74 & 16990 & 17.61 \\
\hline Poly(isoprene) & $1 \mathrm{CH}_{3}+2 \mathrm{CH}_{2}+1(=\mathrm{CH})+1(=\mathrm{C})$ & 0.910 & 74.73 & 23210 & 17.62 \\
\hline Poly(vinyl cyclohexane) & $1(6-\operatorname{ring})+6 \mathrm{CH}_{2}+2 \mathrm{CH}$ & 0.805 & 136.65 & 37550 & 16.58 \\
\hline Poly(vinyl 2-pyridine) & $1 \mathrm{CH}_{2}+1 \mathrm{CH}+1(-\mathrm{N}=\mathrm{C})+6(\mathrm{C}=\mathrm{C})$ & 0.977 & 107.47 & 37230 & 18.61 \\
\hline Poly(vinyl 4-pyridine) & $1 \mathrm{CH}_{2}+1 \mathrm{CH}+1(-\mathrm{N}=\mathrm{C})+6(\mathrm{C}=\mathrm{C})$ & 0.988 & 106.28 & 37230 & 18.71 \\
\hline Poly(oxybutylene) & $4 \mathrm{CH}_{2}+10$ & 0.970 & 74.23 & 23110 & 17.64 \\
\hline $\begin{array}{l}\text { Poly }(2,5 \text {-bis }[(4- \\
\text { butoxyphenyl) } \\
\text { oxycarbonyl] styrene) } \\
\text { (PBPCS) }\end{array}$ & $\begin{array}{l}2 \mathrm{CH}_{2}+7 \mathrm{CH}_{2}+1 \mathrm{CH}+2 \mathrm{COO}+2 \mathrm{O}+ \\
3 \text { (phenyl) }\end{array}$ & 1.250 & 390.40 & 185950 & 21.82 \\
\hline Poly(methyl methacrylate) & $2 \mathrm{CH}_{3}+1 \mathrm{CH}_{2}+1 \mathrm{COO}+1(>\mathrm{C}<)$ & 1.180 & 84.75 & 33830 & 19.98 \\
\hline $\begin{array}{l}\text { Poly(ethyl hexyl } \\
\text { methacrylate) }\end{array}$ & $\begin{array}{l}3 \mathrm{CH}_{3}+6 \mathrm{CH}_{2}+1 \mathrm{CH}+1 \mathrm{COO}+1(>\mathrm{C}< \\
)\end{array}$ & 0.908 & 218.06 & 67550 & 17.60 \\
\hline Poly(butyl methacrylate) & $2 \mathrm{CH}_{3}+4 \mathrm{CH}_{2}+1 \mathrm{COO}+1(>\mathrm{C}<)$ & 1.045 & 136.00 & 48650 & 18.91 \\
\hline Poly(oxypropylene) & $1 \mathrm{CH}_{3}+1 \mathrm{CH}_{2}+1 \mathrm{CH}+10$ & 1.030 & 56.32 & 16430 & 17.08 \\
\hline Poly(t-butylacrylate) & $\begin{array}{l}1\left(=\mathrm{CH}_{2}\right)+1(\mathrm{COO})+1(>\mathrm{C}<)+ \\
3\left(\mathrm{CH}_{3}\right)\end{array}$ & 1.000 & 128.20 & 37910 & 17.20 \\
\hline Poly(alphamethyl styrene) & $1 \mathrm{Ph}+1 \mathrm{CH}_{3}+1\left(=\mathrm{CH}_{2}\right)+1(>\mathrm{C}=)$ & 0.910 & 129.87 & 45270 & 18.67 \\
\hline Poly(propylene carbonate) & $\begin{array}{l}1 \mathrm{CO}_{2}+1(-\mathrm{O}-)+1(\mathrm{CH})+1\left(\mathrm{CH}_{2}\right)+ \\
1\left(\mathrm{CH}_{3}\right)\end{array}$ & 1.204 & 84.72 & 34430 & 20.16 \\
\hline Poly(dihydroxy styrene) & $1(\mathrm{Ph}-\mathrm{p})+1 \mathrm{CH}_{2}+1 \mathrm{CH}_{3}+2(\mathrm{OH})$ & 1.160 & 119.83 & 101190 & 29.06 \\
\hline Poly(4-hyrdoxy styrene) & $\begin{array}{l}1(\mathrm{Ph})+1(>\mathrm{CH}-)+1\left(-\mathrm{CH}_{2}-\right)+ \\
1(\mathrm{OH})\end{array}$ & 1.160 & 103.44 & 70110 & 26.03 \\
\hline $\begin{array}{l}\text { Poly(styrene sulfonate) } \\
\text { (PSS) }\end{array}$ & $\begin{array}{l}1(\mathrm{Ph})+1(\text { Phenylene })+1\left(\mathrm{CH}_{3}\right)+ \\
2\left(\mathrm{CH}_{2}\right)+1(\mathrm{CH})+1 \mathrm{SO}_{3}\end{array}$ & 1.245 & 232.13 & 100740 & 20.83 \\
\hline $\begin{array}{l}\text { Poly (2-methyl-1,3- } \\
\text { pentadiene) (P2MP) }\end{array}$ & $3\left(\mathrm{CH}_{3}\right)+1(>\mathrm{C}=)+1(\mathrm{CH}=)+1 \mathrm{CH}_{2}$ & 0.900 & 93.33 & 27690 & 17.22 \\
\hline $\begin{array}{l}\text { Polyoligo(ethylene glycol) } \\
\text { methyl ether methacrylate } \\
\text { (POEGMA) }\end{array}$ & $\begin{array}{l}1\left(\mathrm{CH}_{3}\right)+1(\mathrm{CH})+1\left(\mathrm{CO}_{2}\right)+2\left(\mathrm{CH}_{2}\right)+ \\
1(-\mathrm{O}-)\end{array}$ & 1.101 & 105.36 & 39370 & 19.33 \\
\hline $\begin{array}{l}\text { Polyethylene-co-polyethyl } \\
\text { ethylene }\end{array}$ & $4\left(\mathrm{CH}_{2}\right)+\mathrm{CH}_{3}+\mathrm{CH}$ & 0.925 & 90.81 & 29640 & 18.07 \\
\hline Poly-pdmb-precursor & $\begin{array}{l}2(-\mathrm{O}-)+1(\mathrm{Ph}-\mathrm{p})+1\left(\mathrm{CH}_{2}=\right)+ \\
3(\mathrm{CH}=)+3(\mathrm{CH})+1\left(\mathrm{CH}_{2}\right)\end{array}$ & 1.000 & $214 / 1$ & 71110 & 18.23 \\
\hline $\begin{array}{l}\text { Poly }[(\text { trimethylsiloxy)- } \\
\text { methyl)norbomene }]^{4}\end{array}$ & $\begin{array}{l}1(5-\text { ring })+3 \mathrm{CH}_{2}+2(\mathrm{CH})+ \\
1(\mathrm{CH}=)+1\left(\mathrm{CH}_{2}=\right)+1\left(\mathrm{CH}_{2}\right)+ \\
1(-0-)+1 \mathrm{Si}\end{array}$ & 1.000 & $153 / 1$ & 21350 & 11.81 \\
\hline
\end{tabular}




\section{Dataset Statistics}

(a)

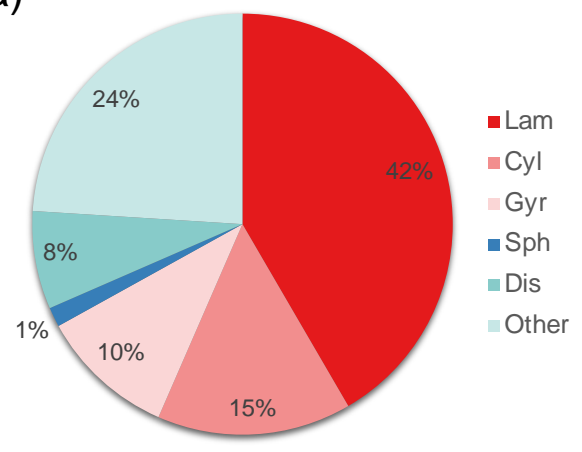

(b)

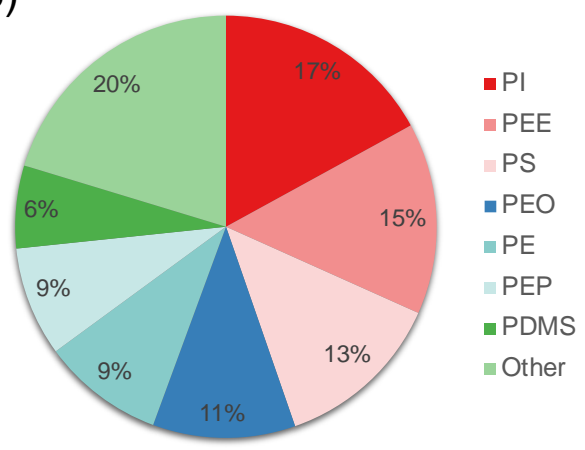

Figure S1: Data statistics: (a) distribution of pure phases and (b) distribution of chemistries within the dataset. In panel (a), the "other" category includes phase transitions. These statistics are computed from the complete dataset reported in Ref. 41 of the main manuscript, which includes the perforated and $F d d d\left(\mathrm{O}^{70}\right)$ network phases, collectively denoted as "other". Since the number of datapoints for such other phases are very few, the above statistics are almost an exact representation of the dataset used in this manuscript (which does not include perforated and $F d d d\left(\mathrm{O}^{70}\right)$ network phases).

\section{Machine Learning Model Description}

\subsection{Random Forest Model Setup}

In this work, a Random Forest Classifier (RFC) is used to model the non-linear relationship between the features governing the self-assembly of block copolymers and the self-assembled nanostructure. The phase behavior of $\mathrm{AB}$ diblock copolymers is modeled by incorporating the following five features: temperature $(T)$, volume fraction of block A $\left(f_{A}\right)$, total molar mass $\left(M_{n}\right)$, and the blocks' chemical identities. The effect of conformational asymmetry, i.e., the ratio of blocks Kuhn lengths $\left(b_{A} / b_{B}\right)$ is assumed to be captured by incorporating the blocks' chemical identities instead of using $b_{A} / b_{B}$ as an explicit feature. For the cases in which block identities are not used as explicit features, conformational asymmetry is neglected. Additionally, the calculation of volume fraction $\left(f_{A}\right)$ depends on blocks densities $\left(\rho_{A}\right.$ and $\left.\rho_{B}\right)$ which further depends on temperature; however, such a subtle variation is neglected in this work. For each sample in the dataset, the input vector is formed by concatenating the numerical values of overall molar mass of polymer $\left(M_{n}\right)$, volume fraction $\left(f_{A}\right)$, and temperature $(T)$, and descriptors or fingerprints for both the blocks. Several different methods are used to create molecular fingerprints, as described earlier. For the case with no descriptors, only $\left(M_{n}, f_{A}, T\right)$ represents the complete input feature set.

For each entry in the dataset, the fingerprint vectors for the individual blocks are generated separately and concatenated with each other as well as with the respective values of $T, f_{A}$, and $M_{n}$ to constitute the complete feature set used to describe the block copolymer. In principle, the order of concatenation of individual blocks vectors, i.e., concatenation of vectors for $\mathrm{AB}$ versus $\mathrm{BA}$, should not have any effect on the machine-learning model. To incorporate such symmetry into the model, a new datapoint is created by modifying the order of blocks as $\mathrm{BA}$ and volume fraction as $1-f_{A}$, corresponding to each datapoint represented as $\mathrm{AB}$ in the original dataset. This essentially doubles the original dataset, and this doubleddataset is then used to develop the machine-learning model. 
The output phase for each data set is denoted as a one-hot encoded vector that represents the phases in the following order: (Lam, Cyl, Sph, Gyr, Dis). For example, the Lam phase is represented as $y=(1,0,0,0,0)$, while the Lam-Dis transition is represented as $(1,0,0,0,1)$. In this one-hot encoded representation, the complete problem can be viewed as a multi-label classification problem. During the model development, the data is first shuffled, and $75 \%$ of the data is used for training while $25 \%$ is reserved as a test set. Additionally, since the data is highly imbalanced, containing disproportionate number of data points for each phase, the split into training and test sets is performed in a stratified manner, i.e., by selecting the training and test data points in $75 \%-25 \%$ proportion exclusively for each phase.

\subsection{Performance Metrics}

The performance of SCFT and RFC models is assessed by computing the following two accuracy metrics:

$$
\begin{gathered}
\text { Accuracy }=\frac{1}{N} \sum_{i=1}^{N} I\left(y_{i}^{t}, y_{i}^{p}\right), \\
1-(\text { Hamming loss })=1-\frac{1}{N \times L} \sum_{i=1}^{N} \sum_{j=1}^{L} \operatorname{XOR}\left(y_{i, j}^{t}, y_{i, j}^{p}\right),
\end{gathered}
$$

where $y^{t}$ and $y^{p}$ are the true and predicted outputs (observed phases), $N$ is the number of data points (samples), and $L$ is the number of phases. As mentioned earlier, the output $y$ is a vector of length 5 , representing a multi-label one-hot encoded output for the observed phase in the following format: (Lam, Cyl, Sph, Gyr, Dis) or $y=(1,0,0,0,1)$ representing a Lam-dis transition. The function XOR in the above equations is an exclusive-or function with the following values: $\operatorname{XOR}(x, y)=0$ if $x=y$ else $\operatorname{XOR}(x, y)=1$, and the function $I(x, y)$ is the identity function having the values, $I(x, y)=1$ if $x=y$ else $I(x, y)=0$. Note that in Eq. (3), XOR is applied to the whole vector $y_{i}$, while in Eq. (4) it is applied to individual vector components, taking into account the partial correctness (any one of the two phases is correct) of the phase transition points.

\subsection{Fingerprinting Methods}

Chemical molecules must be transformed to their respective numerical representations before a machinelearning algorithm can be used. This transformation step is commonly referred to as fingerprinting in materials informatics. This work uses several fingerprinting methods that are well-established for modeling structure-property relationships for small molecules. In addition, a few fundamental fingerprinting schemes called Singlets, Doublets, and Triplets ${ }^{5}$ that provide information about local atoms, groups and their connectivity are also considered.

For each polymer in the dataset, the singlets, doublets, and triplets are generated using an infinitely long polymer representation, encoded using BigSMILES. The polymer's repeat unit is made into a graph representation, including the connector type (' $\$$ ' or ' $<$ ' or ' $>$ '). From this graph representation, all the singlets are found by visiting each node of the graph. Each singlet is specified by four properties: the atom type, hybridization (either 'sp3', 'sp2' or 'sp1'), the number of non-hydrogen atoms it is bonded to, and its position (backbone or side chain). Using the list of all the possible singlets, the list of doublets is generated. 
A double (triplet) consists of two (three) singlets that have a covalent bond between them specified by one of the following connectors: ", '=' or ' $:$ '. For doublets and triplets, the nodes and edges of the graph are scanned through to determine the bond type between atoms. To count the bonds between repeat units, the tacticity of the polymer is assumed to be all head-to-tail. The tuple vector starts as a vector of zeros of the length of the feature vector. For each tuple that occurs in the polymer, a count is added to the corresponding tuple's index. The final tuples vector of singlets, doublets and triplets is the occurrence count of each tuple divided by the total number of non-hydrogen atoms in a repeat unit of the polymer.

Once all the polymers have been vectorized in this way, a tuples vector matrix is generated with each column corresponding to a polymer of the dataset and each row corresponding to a tuple. The last step is the reduction of the matrix by removing any row that consists of only zeros (meaning this tuple did not occur in any of the polymers of the dataset). A corresponding string format list of the tuples is generated, which keeps the same ordering as the tuples matrix.

The tuples are generated using the code (BigSMILES_vectorization.py) provided in the Github repository: https://github.com/olsenlabmit/BigSMILEStoTuples. The list of non-hydrogen atoms present in the dataset are specified as input to the tuples-generation code, and all possible singlet, doublet and triplet formed by these atoms are output into respective feature vectors. Currently, the only atoms that can be present in the polymer dataset are $\mathrm{C}, \mathrm{c}, \mathrm{N}, \mathrm{n}, \mathrm{O}, \mathrm{o}, \mathrm{Si}, \mathrm{S}, \mathrm{F}, \mathrm{Cl}, \mathrm{Br}$ (lower-case letters representing aromatic atoms). The code should only be used for linear polymers. Repeat units that have more than one connector would lead to improper counting of the bonds that connect different repeat units. The code is currently only effective for polymers with a single repeat unit. Moreover, this vectorization scheme cannot account for specific stereoisomerism.

Below is a brief description for each type of fingerprinting method used in this work:

1. No-chem: This refers to the case when no fingerprints/descriptors are used, and the machine-learning model is formed by using $T, f_{A}$, and $M_{n}$ as the only features. As mentioned earlier, this case neglects the effects of conformational asymmetry and density variation between blocks.

2. GCT: This refers to the case where $\chi_{A B}$ calculated using the GCT method (Section 1) is used as a chemical descriptor/feature for developing the machine learning model. Similar to the previous case, the effects of conformational asymmetry and density variation between blocks are neglected in this case.

3. Singlets: A normalized one-hot encoded vector of all the heavy atoms present in the molecule is used as the descriptor. Hybridization states of each atom are differentiated. For example, $\mathrm{sp}^{2}$ hybridized $\mathrm{C}$ $(=\mathrm{CH} 2)$ is considered different than $\mathrm{sp}^{3}$ hybridized $\mathrm{C}(-\mathrm{CH} 3)$. For all the blocks chemistries considered in this work, the number of distinct singlets is 22 .

4. Doublets: A normalized one-hot encoded vector of substructures formed by pairs of two bonded heavy atoms is used as the chemical representation. The number of distinct doublets for the data set is 52 .

5. Triplets: A normalized one-hot encoded vector of substructures formed by triplets of three bonded heavy atoms. The number of distinct triplets is 124 .

6. Morgan fingerprint (Morgan-fp): This is a commonly-used fingerprint type in cheminformatics and drug discovery. This method generates a specified-sized vector containing binary values, with each value indicating the presence (1) or absence (0) of a specific substructure in the molecule. A list of possible substructures is formed iteratively, beginning from a single atom, and including its bonded 
neighbors at successive iterations. ${ }^{6}$ Accordingly, the number of iterations and the length of the vector are the hyperparameters for this fingerprint.

7. RDKit topological fingerprint (RDKit-fp): This is similar to Morgan fingerprint, with the key difference being the procedure to generate a list of substructures. This fingerprint follows a path-based approach, in which substructures are generated by recording the groups as they appear along the path starting from one end of a molecule and terminating at the other end. ${ }^{7}$ The minimum and maximum paths, along with the fingerprint length, constitute the set of hyperparameters for this fingerprint.

8. RDKit descriptors (RDKit-desc): This method generates a set of values of 200 molecular properties, such as topological polar surface area, number of $\mathrm{NHOH}$ groups, number of aromatic rings, etc. A complete list of the properties is given in the supporting information of Ref. ${ }^{8}$.

The Morgan-fp, RDKit-fp, and RDKit-desc are generated using a SMILES representation for a finite-size polymer chain. In order to capture the high-molecular weight nature of polymers and eliminate endgroup effects, a periodic boundary condition is applied by joining the first and last atoms in the repeat unit (in SMILES representation) to form an oligomeric ring where the number of repeat units acts as a hyperparameter. Figure S2(a) plots the accuracy for various number of repeat units within the polymer chain used for generating the fingerprint vector. It is observed that once the SMILES repeat unit is made as a ring polymer, the number of units within the chain does not have any noticeable effect on the model performance; the accuracy varies by less than $1 \%$ as number of repeat unit changes.

In addition to the number of repeat units, the results may also depend on the length of the fingerprint vector. Figure S2(b) plots the accuracy as a function of fingerprint length. Surprisingly, the performance is independent of the length of the fingerprint vector, and fingerprints of length as small as $2^{6}$ also yield good accuracy.
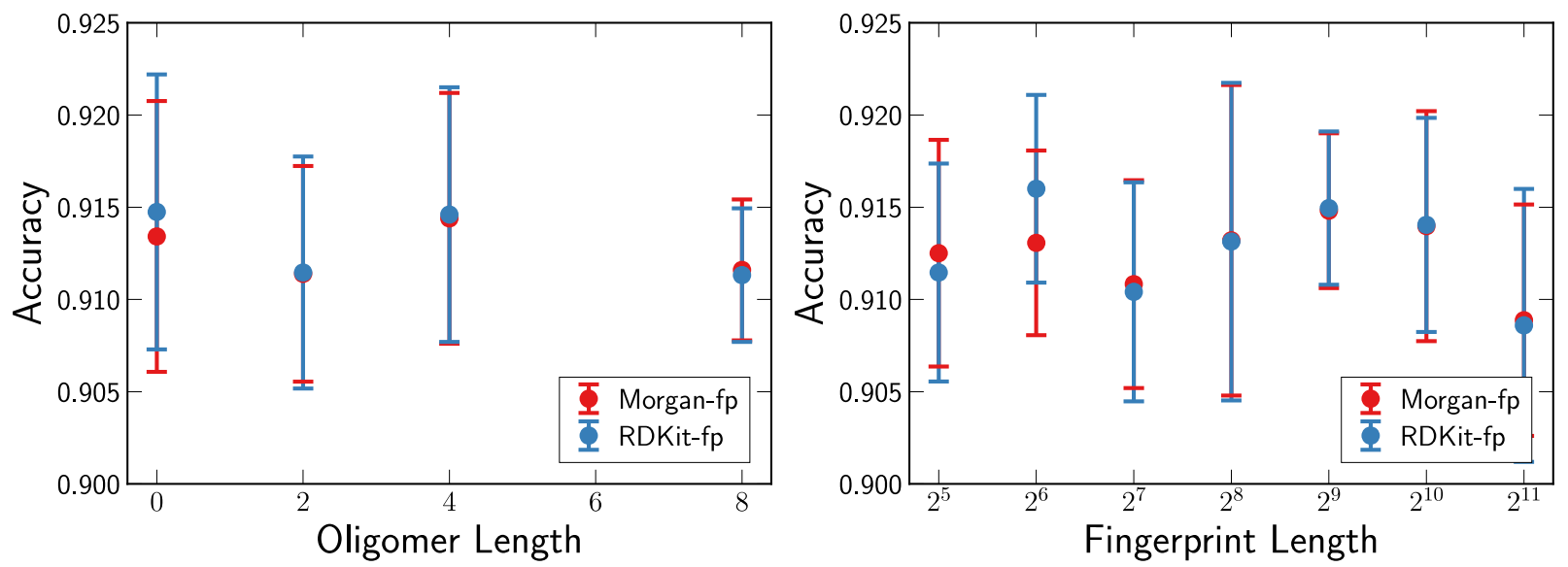

Figure S2: RFC-model accuracies for various number of repeat units in the oligomer SMILES representation that is used to generate the fingerprints. For all the cases, the fingerprint vectors are 1024 bit long. (b) RFC-model accuracies for various lengths of the fingerprint vector, with number of repeat units being 8 for all cases. For both (a) and (b), the radius for Morgan-fp is 5, and the minimum and maximum paths used for RDKit-fp are 3 and 10, respectively.

Table S2: Unique singlets for all the block copolymer chemistries studied in this work. Each singlet is denoted by a set of six characteristics: heavy atom, hybridization, number of hydrogens atoms attached, 
number of non-hydrogen neighbors, and its position (backbone denoted by 'bb', and side group denoted by 'sg'). The full list of singlets is generated using all the heavy atoms as mentioned earlier, while the list used for modeling is a reduced list formed by discarding the singlets that do not exist in the dataset. Below is the reduced list of singlets.

\begin{tabular}{|c|c|}
\hline Number & Tuple \\
\hline 1 & ['C', 'sp3', 3, 1, 'sg'] \\
\hline 2 & ['C', 'sp3', 2, 2, 'bb'] \\
\hline 3 & ['C', 'sp3', 2, 2, 'sg'] \\
\hline 4 & ['C', 'sp3', 1, 3, 'bb'] \\
\hline 5 & ['C', 'sp3', 1, 3, 'sg'] \\
\hline 6 & ['C', 'sp3', 0, 4, 'bb'] \\
\hline 7 & ['C', 'sp3', 0, 4, 'sg'] \\
\hline 8 & ['C', 'sp2', 2, 1, 'sg'] \\
\hline 9 & ['C', 'sp2', 1, 2, 'bb'] \\
\hline 10 & ['C', 'sp2', 1, 2, 'sg'] \\
\hline 11 & ['C', 'sp2', 0, 3, 'bb'] \\
\hline 12 & ['C', 'sp2', 0, 3, 'sg'] \\
\hline 13 & ['c', 'sp2', 1, 2, 'sg'] \\
\hline 14 & ['c', 'sp2', 0, 3, 'sg'] \\
\hline 15 & ['n', 'sp2', 0, 2, 'sg'] \\
\hline 16 & ['O', 'sp3', 1, 1, 'sg'] \\
\hline 17 & ['O', 'sp3', 0, 2, 'bb'] \\
\hline 18 & ['O', 'sp3', 0, 2, 'sg'] \\
\hline 19 & ['O', 'sp2', 0, 1, 'sg'] \\
\hline 20 & ['Si', 'sp3', 0, 4, 'bb'] \\
\hline 21 & ['Si', 'sp3', 0, 4, 'sg'] \\
\hline 22 & ['S', 'sp3d3', 0, 4, 'sg'] \\
\hline
\end{tabular}

Table S3: Unique doublets for all the block copolymer chemistries studied in this work. This is a reduced list of doublets formed by discarding the doublets (from the complete list) that do not exist in the database.

\begin{tabular}{|c|c|}
\hline Number & Tuple \\
\hline 1 & [['C', 'sp3', 3, 1, 'sg'], '-', ['C', 'sp3', 2, 2, 'sg']]] \\
\hline 2 & {$\left[\left[\mathrm{C}^{\prime}\right.\right.$, 'sp3', 3, 1, 'sg'], '-', ['C', 'sp3', 1, 3, 'bb']] } \\
\hline 3 & [['C', 'sp3', 3, 1, 'sg'], '-', ['C', 'sp3', 0, 4, 'bb']] \\
\hline 4 & [['C', 'sp3', 3, 1, 'sg'], '-', ['C', 'sp3', 0, 4, 'sg']]] \\
\hline 5 & [['C', 'sp3', 3, 1, 'sg'], '-', ['C', 'sp2', 0, 3, 'bb']] \\
\hline 6 & {$\left[\left[\mathrm{C}^{\prime}\right.\right.$, 'sp3', 3, 1, 'sg'], '-', ['O', 'sp3', 0, 2, 'sg']] } \\
\hline 7 & [['C', 'sp3', 3, 1, 'sg'], '-', ['Si', 'sp3', 0, 4, 'bb']] \\
\hline 8 & {[]$^{[} \mathrm{C}^{\prime}$, 'sp3', 3, 1, 'sg'], '-', ['Si', 'sp3', 0, 4, 'sg']] } \\
\hline 9 & [['C', 'sp3', 2, 2, 'bb'], '-', ['C', 'sp3', 2, 2, 'bb']] \\
\hline
\end{tabular}




\begin{tabular}{|c|c|}
\hline 10 & [['C', 'sp3', 2, 2, 'bb'], '-', ['C', 'sp3', 1, 3, 'bb']] \\
\hline 11 & [['C', 'sp3', 2, 2, 'bb'], '-', ['C', 'sp3', 0, 4, 'bb']] \\
\hline 12 & [['C', 'sp3', 2, 2, 'bb'], '-', ['C', 'sp2', 1, 2, 'bb']] \\
\hline 13 & [['C', 'sp3', 2, 2, 'bb'], '-', ['C', 'sp2', 0, 3, 'bb']] \\
\hline 14 & [['C', 'sp3', 2, 2, 'bb'], '-', ['O', 'sp3', 0, 2, 'bb']] \\
\hline 15 & [['C', 'sp3', 2, 2, 'sg'], '-', ['C', 'sp3', 2, 2, 'sg']] \\
\hline 16 & [['C', 'sp3', 2, 2, 'sg'], '-', ['C', 'sp3', 1, 3, 'bb']] \\
\hline 17 & [['C', 'sp3', 2, 2, 'sg'], '-', ['C', 'sp3', 1, 3, 'sg']] \\
\hline 18 & {[]$^{\prime} \mathrm{C}$ ', 'sp3', 2, 2, 'sg'], '-', ['O', 'sp3', , 2, 'sg']] } \\
\hline 19 & [['C', 'sp3', 1, 3, 'bb'], '-', ['C', 'sp3', 1, 3, 'bb']] \\
\hline 20 & 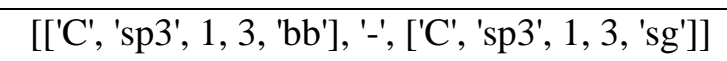 \\
\hline 21 & [['C', 'sp3', 1, 3, 'bb'], '-', ['C', 'sp2', 1, 2, 'bb']] \\
\hline 22 & [['C', 'sp3', 1,3, 'bb'], '-', ['C', 'sp2', 1, 2, 'sg']] \\
\hline 23 & [['C', 'sp3', 1, 3, 'bb'], '-', ['C', 'sp2', 0, 3, 'bb']] \\
\hline 24 & 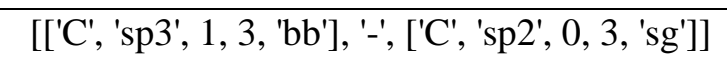 \\
\hline 25 & [['C', 'sp3', 1, 3, 'bb'], '-', ['c', 'sp2', 0, 3, 'sg']] \\
\hline 26 & [['C', 'sp3', 1, 3, 'bb'], '-', ['O', 'sp3', , , 2, 'bb']] \\
\hline 27 & [['C', 'sp3', 1, 3, 'sg'], '-', ['C', 'sp2', 1, 2, 'sg']] \\
\hline 28 & [['C', 'sp3', 1, 3, 'sg'], '-', ['c', 'sp2', 0, 3, 'sg']] \\
\hline 29 & [['C', 'sp3', 0, 4, 'bb'], '-', ['C', 'sp2', , 3, 'sg']] \\
\hline 30 & [['C', 'sp3', 0, 4, 'bb'], '-', ['c', 'sp2', 0, 3, 'sg']] \\
\hline 31 & [['C', 'sp3', 0, 4, 'sg'], '-', ['O', 'sp3', 0, 2, 'sg']] \\
\hline 32 & [['C', 'sp2', 2, 1, 'sg'], '=', ['C', 'sp2', 1, 2, 'sg']] \\
\hline 33 & [['C', 'sp2', 1, 2, 'bb'], '=', ['C', 'sp2', 1, 2, 'bb']] \\
\hline 34 & 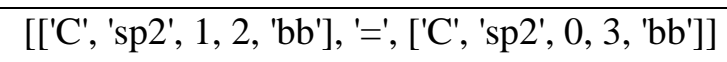 \\
\hline 35 & [['C', 'sp2', 1, 2, 'sg'], '=', ['C', 'sp2', 1, 2, 'sg']] \\
\hline 36 & [['C', 'sp2', 0, 3, 'bb'], '-', ['O', 'sp3', 0, 2, 'bb']] \\
\hline 37 & [['C', 'sp2', 0, 3, 'bb'], '=', ['O', 'sp2', 0, 1, 'sg']] \\
\hline 38 & [['C', 'sp2', 0, 3, 'sg'], '-', ['c', 'sp2', 0, 3, 'sg']] \\
\hline 39 & [['C', 'sp2', 0, 3, 'sg'], '-', ['O', 'sp3', 0, 2, 'sg']] \\
\hline 40 & [['C', 'sp2', 0, 3, 'sg'], '=', ['O', 'sp2', 0, 1, 'sg']] \\
\hline 41 & [['c', 'sp2', 1, 2, 'sg'], ':', ['c', 'sp2', 1, 2, 'sg']] \\
\hline 42 & [['c', 'sp2', 1, 2, 'sg'], ':', ['c', 'sp2', 0, 3, 'sg']] \\
\hline 43 & [['c', 'sp2', 1, 2, 'sg'], ':', ['n', 'sp2', 0, 2, 'sg']] \\
\hline 44 & [['c', 'sp2', 0, 3, 'sg'], ':', ['c', 'sp2', 0, 3, 'sg']] \\
\hline 45 & [['c', 'sp2', 0, 3, 'sg'], ':', ['n', 'sp2', 0, 2, 'sg']] \\
\hline 46 & [['c', 'sp2', 0, 3, 'sg'], '-', ['O', 'sp3', 1, 1, 'sg']] \\
\hline 47 & [['c', 'sp2', 0, 3, 'sg'], '-', ['O', 'sp3', 0, 2, 'sg']] \\
\hline 48 & {$\left[\left[\mathrm{c}^{\prime}\right.\right.$, 'sp2', 0,3, , 'sg'], '-', ['S', 'sp3d3', 0, 4, 'sg']] } \\
\hline
\end{tabular}




\begin{tabular}{|c|c|}
\hline 49 & [['O', 'sp3', 1, 1, 'sg'], '-', ['S', 'sp3d3', 0, 4, 'sg']] \\
\hline 50 & [['O', 'sp3', 0, 2, 'bb'], '-', ['Si', 'sp3', 0, 4, 'bb']] \\
\hline 51 & [['O', 'sp3', 0, 2, 'sg'], '-', ['Si', 'sp3', 0, 4, 'sg']] \\
\hline 52 & 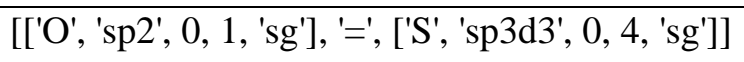 \\
\hline
\end{tabular}

Table S4: Unique triplets for all the block copolymer chemistries studied in this work. This is a reduced list of triplets formed by discarding the triplets (from the complete list) that do not exist in the database.

\begin{tabular}{|c|c|}
\hline Number & 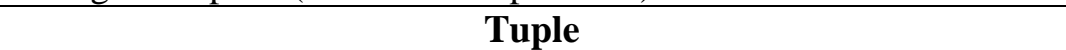 \\
\hline 1 & [['C', 'sp3', 2, 2, 'bb'], '-', ['C', 'sp3', 2, 2, 'bb'], '-', ['C', 'sp3', 2, 2, 'bb']] \\
\hline 2 & [['C', 'sp3', 2, 2, 'bb'], '-', ['C', 'sp3', 2, 2, 'bb'], '-', ['C', 'sp3', 1, 3, 'bb']] \\
\hline 3 & [['C', 'sp3', 2, 2, 'bb'], '-', ['C', 'sp3', 2, 2, 'bb'], '-', ['C', 'sp3', 0, 4, 'bb']] \\
\hline 4 & [['C', 'sp3', 2, 2, 'bb'], '-', ['C', 'sp3', 2, 2, 'bb'], '-', ['C', 'sp2', 1, 2, 'bb']] \\
\hline 5 & [['C', 'sp3', 2, 2, 'bb'], '-', ['C', 'sp3', 2, 2, 'bb'], '-', ['C', 'sp2', 0, 3, 'bb']] \\
\hline 6 & [['C', 'sp3', 2, 2, 'bb'], '-', ['C', 'sp3', 2, 2, 'bb'], '-', ['O', 'sp3', 0, 2, 'bb']] \\
\hline 7 & [['C', 'sp3', 1, 3, 'bb'], '-', ['C', 'sp3', 2, 2, 'bb'], '-', ['C', 'sp3', 1, 3, 'bb']] \\
\hline 8 & [['C', 'sp3', 1, 3, 'bb'], '-', ['C', 'sp3', 2, 2, 'bb'], '-', ['C', 'sp2', 0, 3, 'bb']] \\
\hline 9 & [['C', 'sp3', 1, 3, 'bb'], '-', ['C', 'sp3', 2, 2, 'bb'], '-', ['O', 'sp3', 0, 2, 'bb']] \\
\hline 10 & [['C', 'sp3', 0, 4, 'bb'], '-', ['C', 'sp3', 2, 2, 'bb'], '-', ['C', 'sp3', 0, 4, 'bb']] \\
\hline 11 & [['C', 'sp3', 3, 1, 'sg'], '-', ['C', 'sp3', 2, 2, 'sg'], '-', ['C', 'sp3', 2, 2, 'sg']] \\
\hline 12 & [['C', 'sp3', 3, 1, 'sg'], '-', ['C', 'sp3', 2, 2, 'sg'], '-', ['C', 'sp3', 1, 3, 'bb']] \\
\hline 13 & [['C', 'sp3', 3, 1, 'sg'], '-', ['C', 'sp3', 2, 2, 'sg'], '-', ['C', 'sp3', 1, 3, 'sg']] \\
\hline 14 & [['C', 'sp3', 2, 2, 'sg'], '-', ['C', 'sp3', 2, 2, 'sg'], '-', ['C', 'sp3', 2, 2, 'sg']] \\
\hline 15 & [['C', 'sp3', 2, 2, 'sg'], '-', ['C', 'sp3', 2, 2, 'sg'], '-', ['C', 'sp3', 1, 3, 'bb']] \\
\hline 16 & [['C', 'sp3', 2, 2, 'sg'], '-', ['C', 'sp3', 2, 2, 'sg'], '-', ['C', 'sp3', 1, 3, 'sg']] \\
\hline 17 & [['C', 'sp3', 2, 2, 'sg'], '-', ['C', 'sp3', 2, 2, 'sg'], '-', ['O', 'sp3', 0, 2, 'sg']] \\
\hline 18 & [['C', 'sp3', 1, 3, 'bb'], '-', ['C', 'sp3', 2, 2, 'sg'], '-', ['C', 'sp3', 1, 3, 'sg']] \\
\hline 19 & [['C', 'sp3', 1, 3, 'sg'], '-', ['C', 'sp3', 2, 2, 'sg'], '-', ['O', 'sp3', 0, 2, 'sg']] \\
\hline 20 & [['C', 'sp3', 3, 1, 'sg'], '-', ['C', 'sp3', 1, 3, 'bb'], '-', ['C', 'sp3', 2, 2, 'bb']] \\
\hline 21 & [['C', 'sp3', 3, 1, 'sg'], '-', ['C', 'sp3', 1, 3, 'bb'], '-', ['C', 'sp3', 1, 3, 'bb']] \\
\hline 22 & [['C', 'sp3', 3, 1, 'sg'], '-', ['C', 'sp3', 1, 3, 'bb'], '-', ['C', 'sp2', 1, 2, 'bb']] \\
\hline 23 & [['C', 'sp3', 3, 1, 'sg'], '-', ['C', 'sp3', 1, 3, 'bb'], '-', ['C', 'sp2', 0, 3, 'bb']] \\
\hline 24 & [['C', 'sp3', 3, 1, 'sg'], '-', ['C', 'sp3', 1, 3, 'bb'], '-', ['O', 'sp3', 0, 2, 'bb']] \\
\hline 25 & [['C', 'sp3', 2, 2, 'bb'], '-', ['C', 'sp3', 1, 3, 'bb'], '-', ['C', 'sp3', 2, 2, 'bb']] \\
\hline 26 & [['C', 'sp3', 2, 2, 'bb'], '-', ['C', 'sp3', 1, 3, 'bb'], '-', ['C', 'sp3', 2, 2, 'sg']] \\
\hline 27 & [['C', 'sp3', 2, 2, 'bb'], '-', ['C', 'sp3', 1, 3, 'bb'], '-', ['C', 'sp3', 1, 3, 'bb']] \\
\hline 28 & [['C', 'sp3', 2, 2, 'bb'], '-', ['C', 'sp3', 1, 3, 'bb'], '-', ['C', 'sp3', 1, 3, 'sg']] \\
\hline 29 & [['C', 'sp3', 2, 2, 'bb'], '-', ['C', 'sp3', 1, 3, 'bb'], '-', ['C', 'sp2', 1, 2, 'bb']] \\
\hline 30 & [['C', 'sp3', 2, 2, 'bb'], '-', ['C', 'sp3', 1, 3, 'bb'], '-', ['C', 'sp2', 1, 2, 'sg']] \\
\hline 31 & [['C', 'sp3', 2, 2, 'bb'], '-', ['C', 'sp3', 1, 3, 'bb'], '-', ['C', 'sp2', 0, 3, 'sg']] \\
\hline
\end{tabular}




\begin{tabular}{|c|c|}
\hline 32 & [['C', 'sp3', 2, 2, 'bb'], '-', ['C', 'sp3', 1, 3, 'bb'], '-', ['c', 'sp2', 0, 3, 'sg']] \\
\hline 33 & [['C', 'sp3', 2, 2, 'bb'], '-', ['C', 'sp3', 1, 3, 'bb'], '-', ['O', 'sp3', 0, 2, 'bb']] \\
\hline 34 & [['C', 'sp3', 2, 2, 'sg'], '-', ['C', 'sp3', 1, 3, 'bb'], '-', ['C', 'sp3', 1, 3, 'bb']] \\
\hline 35 & [['C', 'sp3', 2, 2, 'sg'], '-', ['C', 'sp3', 1, 3, 'bb'], '-', ['C', 'sp2', 1, 2, 'bb']] \\
\hline 36 & [['C', 'sp3', 2, 2, 'sg'], '-', ['C', 'sp3', 1, 3, 'bb'], '-', ['O', 'sp3', 0, 2, 'bb']] \\
\hline 37 & [['C', 'sp3', 1, 3, 'bb'], '-', ['C', 'sp3', 1, 3, 'bb'], '-', ['C', 'sp3', 1, 3, 'sg']] \\
\hline 38 & [['C', 'sp3', 1, 3, 'bb'], '-', ['C', 'sp3', 1, 3, 'bb'], '-', ['C', 'sp2', 1, 2, 'bb']] \\
\hline 39 & [['C', 'sp3', 1, 3, 'bb'], '-', ['C', 'sp3', 1, 3, 'bb'], '-', ['C', 'sp2', 1, 2, 'sg']] \\
\hline 40 & [['C', 'sp3', 1, 3, 'bb'], '-', ['C', 'sp3', 1, 3, 'bb'], '-', ['C', 'sp2', 0, 3, 'sg']] \\
\hline 41 & [['C', 'sp3', 1, 3, 'bb'], '-', ['C', 'sp3', 1, 3, 'bb'], '-', ['c', 'sp2', 0, 3, 'sg']] \\
\hline 42 & [['C', 'sp3', 1, 3, 'sg'], '-', ['C', 'sp3', 1, 3, 'bb'], '-', ['C', 'sp2', 1, 2, 'bb']] \\
\hline 43 & [['C', 'sp2', 0, 3, 'bb'], '-', ['C', 'sp3', 1, 3, 'bb'], '-', ['O', 'sp3', 0, 2, 'bb']] \\
\hline 44 & [['C', 'sp3', 2, 2, 'sg'], '-', ['C', 'sp3', 1, 3, 'sg'], '-', ['C', 'sp3', 2, 2, 'sg']] \\
\hline 45 & [['C', 'sp3', 2, 2, 'sg'], '-', ['C', 'sp3', 1, 3, 'sg'], '-', ['C', 'sp3', 1, 3, 'bb']] \\
\hline 46 & [['C', 'sp3', 1, 3, 'bb'], '-', ['C', 'sp3', 1, 3, 'sg'], '-', ['C', 'sp2', 1, 2, 'sg']] \\
\hline 47 & [['C', 'sp3', 1, 3, 'bb'], '-', ['C', 'sp3', 1, 3, 'sg'], '-', ['c', 'sp2', 0, 3, 'sg']] \\
\hline 48 & [['C', 'sp2', 1, 2, 'sg'], '-', ['C', 'sp3', 1, 3, 'sg'], '-', ['c', 'sp2', 0, 3, 'sg']] \\
\hline 49 & [['C', 'sp3', 3, 1, 'sg'], '-', ['C', 'sp3', 0, 4, 'bb'], '-', ['C', 'sp3', 2, 2, 'bb']] \\
\hline 50 & [['C', 'sp3', 3, 1, 'sg'], '-', ['C', 'sp3', 0, 4, 'bb'], '-', ['C', 'sp3', 0, 4, 'bb']] \\
\hline 51 & [['C', 'sp3', 3, 1, 'sg'], '-', ['C', 'sp3', 0, 4, 'bb'], '-', ['C', 'sp2', 0, 3, 'sg']] \\
\hline 52 & [['C', 'sp3', 3, 1, 'sg'], '-', ['C', 'sp3', 0, 4, 'bb'], '-', ['c', 'sp2', 0, 3, 'sg']] \\
\hline 53 & [['C', 'sp3', 2, 2, 'bb'], '-', ['C', 'sp3', 0, 4, 'bb'], '-', ['C', 'sp3', 2, 2, 'bb']] \\
\hline 54 & [['C', 'sp3', 2, 2, 'bb'], '-', ['C', 'sp3', 0, 4, 'bb'], '-', ['C', 'sp3', 0, 4, 'bb']] \\
\hline 55 & [['C', 'sp3', 2, 2, 'bb'], '-', ['C', 'sp3', 0, 4, 'bb'], '-', ['C', 'sp2', 0, 3, 'sg']] \\
\hline 56 & [['C', 'sp3', 2, 2, 'bb'], '-', ['C', 'sp3', 0, 4, 'bb'], '-', ['c', 'sp2', 0, 3, 'sg']] \\
\hline 57 & [['C', 'sp3', 0, 4, 'bb'], '-', ['C', 'sp3', 0, 4, 'bb'], '-', ['C', 'sp2', 0, 3, 'sg']] \\
\hline 58 & [['C', 'sp3', 0, 4, 'bb'], '-', ['C', 'sp3', 0, 4, 'bb'], '-', ['c', 'sp2', 0, 3, 'sg']] \\
\hline 59 & [['C', 'sp3', 3, 1, 'sg'], '-', ['C', 'sp3', 0, 4, 'sg'], '-', ['C', 'sp3', 3, 1, 'sg']] \\
\hline 60 & 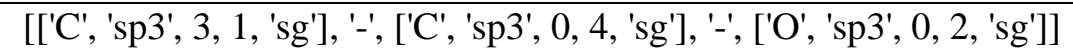 \\
\hline 61 & [['C', 'sp3', 2, 2, 'bb'], '-', ['C', 'sp2', 1, 2, 'bb'], '=', ['C', 'sp2', 1, 2, 'bb']] \\
\hline 62 & [['C', 'sp3', 2, 2, 'bb'], '-', ['C', 'sp2', 1, 2, 'bb'], '=', ['C', 'sp2', 0, 3, 'bb']] \\
\hline 63 & [['C', 'sp3', 1, 3, 'bb'], '-', ['C', 'sp2', 1, 2, 'bb'], '=', ['C', 'sp2', 1, 2, 'bb']] \\
\hline 64 & [['C', 'sp3', 1, 3, 'bb'], '-', ['C', 'sp2', 1, 2, 'bb'], '=', ['C', 'sp2', 0, 3, 'bb']] \\
\hline 65 & [['C', 'sp3', 1, 3, 'bb'], '-', ['C', 'sp2', 1, 2, 'sg'], '=', ['C', 'sp2', 2, 1, 'sg']] \\
\hline 66 & [['C', 'sp3', 1, 3, 'sg'], '-', ['C', 'sp2', 1, 2, 'sg'], '=', ['C', 'sp2', 1, 2, 'sg']] \\
\hline 67 & [['C', 'sp3', 3, 1, 'sg'], '-', ['C', 'sp2', 0, 3, 'bb'], '-', ['C', 'sp3', 2, 2, 'bb']] \\
\hline 68 & [['C', 'sp3', 3, 1, 'sg'], '-', ['C', 'sp2', 0, 3, 'bb'], '=', ['C', 'sp2', 1, 2, 'bb']] \\
\hline 69 & [['C', 'sp3', 2, 2, 'bb'], '-', ['C', 'sp2', 0, 3, 'bb'], '=', ['C', 'sp2', 1, 2, 'bb']] \\
\hline 70 & [['C', 'sp3', 1, 3, 'bb'], '-', ['C', 'sp2', 0, 3, 'bb'], '-', ['O', 'sp3', 0, 2, 'bb']] \\
\hline
\end{tabular}




\begin{tabular}{|c|c|}
\hline 71 & [['C', 'sp3', 1, 3, 'bb'], '-', ['C', 'sp2', 0, 3, 'bb'], '=', ['O', 'sp2', 0, 1, 'sg']] \\
\hline 72 & [['O', 'sp3', 0, 2, 'bb'], '-', ['C', 'sp2', 0, 3, 'bb'], '-', ['O', 'sp3', 0, 2, 'bb']] \\
\hline 73 & [['O', 'sp3', 0, 2, 'bb'], '-', ['C', 'sp2', 0, 3, 'bb'], '=', ['O', 'sp2', 0, 1, 'sg']] \\
\hline 74 & [['C', 'sp3', 1, 3, 'bb'], '-', ['C', 'sp2', 0, 3, 'sg'], '-', ['O', 'sp3', 0, 2, 'sg']] \\
\hline 75 & [['C', 'sp3', 1, 3, 'bb'], '-', ['C', 'sp2', 0, 3, 'sg'], '=', ['O', 'sp2', 0, 1, 'sg']]] \\
\hline 76 & [['C', 'sp3', 0, 4, 'bb'], '-', ['C', 'sp2', 0, 3, 'sg'], '-', ['O', 'sp3', 0, 2, 'sg']] \\
\hline 77 & [['C', 'sp3', 0, 4, 'bb'], '-', ['C', 'sp2', 0, 3, 'sg'], '=', ['O', 'sp2', 0, 1, 'sg']]] \\
\hline 78 & [['c', 'sp2', 0, 3, 'sg'], '-', ['C', 'sp2', 0, 3, 'sg'], '-', ['O', 'sp3', 0, 2, 'sg']] \\
\hline 79 & {$\left[\left[\mathrm{C}^{\prime}\right.\right.$ ', 'sp2', 0, 3, 'sg'], '-', ['C', 'sp2', 0, 3, 'sg'], '=', ['O', 'sp2', 0, 1, 'sg']] } \\
\hline 80 & [['O', 'sp3', 0, 2, 'sg'], '-', ['C', 'sp2', 0, 3, 'sg'], '=', ['O', 'sp2', 0, 1, 'sg']] \\
\hline 81 & [['c', 'sp2', 1, 2, 'sg'], ':', ['c', 'sp2', 1, 2, 'sg'], ':', ['c', 'sp2', 1, 2, 'sg']] \\
\hline 82 & [['c', 'sp2', 1, 2, 'sg'], ':', ['c', 'sp2', 1, 2, 'sg'], ':', ['c', 'sp2', 0, 3, 'sg']] \\
\hline 83 & [['c', 'sp2', 1, 2, 'sg'], ':', ['c', 'sp2', 1, 2, 'sg'], ':', ['n', 'sp2', 0, 2, 'sg']]] \\
\hline 84 & [['c', 'sp2', 0, 3, 'sg'], ':', ['c', 'sp2', 1, 2, 'sg'], ':', ['c', 'sp2', 0, 3, 'sg']]] \\
\hline 85 & [['C', 'sp3', 1, 3, 'bb'], '-', ['c', 'sp2', 0, 3, 'sg'], ':', ['c', 'sp2', 1, 2, 'sg']] \\
\hline 86 & [['C', 'sp3', 1, 3, 'bb'], '-', ['c', 'sp2', 0, 3, 'sg'], ':', ['c', 'sp2', 0, 3, 'sg']] \\
\hline 87 & [['C', 'sp3', 1, 3, 'bb'], '-', ['c', 'sp2', 0, 3, 'sg'], ':', ['n', 'sp2', 0, 2, 'sg']] \\
\hline 88 & [['C', 'sp3', 1, 3, 'sg'], '-', ['c', 'sp2', 0, 3, 'sg'], ':', ['c', 'sp2', 0, 3, 'sg']]] \\
\hline 89 & [['C', 'sp3', 0, 4, 'bb'], '-', ['c', 'sp2', 0, 3, 'sg'], ':', ['c', 'sp2', 1, 2, 'sg']] \\
\hline 90 & [['C', 'sp2', 0, 3, 'sg'], '-', ['c', 'sp2', , 3, 'sg'], ':', ['c', 'sp2', 1, 2, 'sg']] \\
\hline 91 & [['C', 'sp2', 0, 3, 'sg'], '-', ['c', 'sp2', 0, 3, 'sg'], ':', ['c', 'sp2', 0, 3, 'sg']] \\
\hline 92 & [['c', 'sp2', 1, 2, 'sg'], ':', ['c', 'sp2', 0, 3, 'sg'], ':', ['c', 'sp2', 1, 2, 'sg']] \\
\hline 93 & [['c', 'sp2', 1, 2, 'sg'], ':', ['c', 'sp2', 0, 3, 'sg'], ':', ['c', 'sp2', 0, 3, 'sg']]] \\
\hline 94 & [['c', 'sp2', 1, 2, 'sg'], ':', ['c', 'sp2', 0, 3, 'sg'], ':', ['n', 'sp2', 0, 2, 'sg']] \\
\hline 95 & [['c', 'sp2', 1, 2, 'sg'], ':', ['c', 'sp2', 0, 3, 'sg'], '-', ['O', 'sp3', 1, 1, 'sg']] \\
\hline 96 & [['c', 'sp2', 1, 2, 'sg'], ':', ['c', 'sp2', 0, 3, 'sg'], '-', ['O', 'sp3', 0, 2, 'sg']]] \\
\hline 97 & [['c', 'sp2', 1, 2, 'sg'], ':', ['c', 'sp2', 0, 3, 'sg'], '-', ['S', 'sp3d3', 0, 4, 'sg']] \\
\hline 98 & [['c', 'sp2', 0, 3, 'sg'], ':', ['c', 'sp2', 0, 3, 'sg'], ':', ['c', 'sp2', 0, 3, 'sg']] \\
\hline 99 & [['c', 'sp2', 0, 3, 'sg'], ':', ['c', 'sp2', 0, 3, 'sg'], '-', ['O', 'sp3', 1, 1, 'sg']] \\
\hline 100 & [['c', 'sp2', 0, 3, 'sg'], ':', ['c', 'sp2', 0, 3, 'sg'], '-', ['O', 'sp3', 0, 2, 'sg']] \\
\hline 101 & [['c', 'sp2', 1, 2, 'sg'], ':', ['n', 'sp2', 0, 2, 'sg'], ':', ['c', 'sp2', 1, 2, 'sg']] \\
\hline 102 & [['c', 'sp2', 1, 2, 'sg'], ':', ['n', 'sp2', 0, 2, 'sg'], ':', ['c', 'sp2', 0, 3, 'sg']] \\
\hline 103 & [['C', 'sp3', 2, 2, 'bb'], '-', ['O', 'sp3', 0, 2, 'bb'], '-', ['C', 'sp3', 2, 2, 'bb']] \\
\hline 104 & [['C', 'sp3', 2, 2, 'bb'], '-', ['O', 'sp3', 0, 2, 'bb'], '-', ['C', 'sp3', 1, 3, 'bb']] \\
\hline 105 & [['C', 'sp3', 2, 2, 'bb'], '-', ['O', 'sp3', 0, 2, 'bb'], '-', ['C', 'sp2', 0, 3, 'bb']] \\
\hline 106 & [['C', 'sp3', 1, 3, 'bb'], '-', ['O', 'sp3', 0, 2, 'bb'], '-', ['C', 'sp2', 0, 3, 'bb']] \\
\hline 107 & [['Si', 'sp3', 0, 4, 'bb'], '-', ['O', 'sp3', 0, 2, 'bb'], '-', ['Si', 'sp3', 0, 4, 'bb']] \\
\hline 108 & [['C', 'sp3', 3, 1, 'sg'], '-', ['O', 'sp3', 0, 2, 'sg'], '-', ['C', 'sp3', 2, 2, 'sg']] \\
\hline 109 & [['C', 'sp3', 3, 1, 'sg'], '-', ['O', 'sp3', , 2, 'sg'], '-', ['C', 'sp2', 0, 3, 'sg']] \\
\hline
\end{tabular}




\begin{tabular}{|c|c|}
\hline 110 & [['C', 'sp3', 3, 1, 'sg'], '-', ['O', 'sp3', 0, 2, 'sg'], '-', ['c', 'sp2', 0, 3, 'sg']] \\
\hline 111 & [['C', 'sp3', 2, 2, 'sg'], '-', ['O', 'sp3', 0, 2, 'sg'], '-', ['C', 'sp2', 0, 3, 'sg']] \\
\hline 112 & [['C', 'sp3', 2, 2, 'sg'], '-', ['O', 'sp3', 0, 2, 'sg'], '-', ['c', 'sp2', 0, 3, 'sg']] \\
\hline 113 & [['C', 'sp3', 2, 2, 'sg'], '-', ['O', 'sp3', 0, 2, 'sg'], '-', ['Si', 'sp3', 0, 4, 'sg']] \\
\hline 114 & [['C', 'sp3', 0, 4, 'sg'], '-', ['O', 'sp3', 0, 2, 'sg'], '-', ['C', 'sp2', 0, 3, 'sg']] \\
\hline 115 & [['C', 'sp2', 0, 3, 'sg'], '-', ['O', 'sp3', 0, 2, 'sg'], '-', ['c', 'sp2', 0, 3, 'sg']] \\
\hline 116 & [['C', 'sp3', 3, 1, 'sg'], '-', ['Si', 'sp3', 0, 4, 'bb'], '-', ['C', 'sp3', 3, 1, 'sg']] \\
\hline 117 & [['C', 'sp3', 3, 1, 'sg'], '-', ['Si', 'sp3', 0, 4, 'bb'], '-', ['O', 'sp3', 0, 2, 'bb']] \\
\hline 118 & [['O', 'sp3', 0, 2, 'bb'], '-', ['Si', 'sp3', 0, 4, 'bb'], '-', ['O', 'sp3', 0, 2, 'bb']] \\
\hline 119 & [['C', 'sp3', 3, 1, 'sg'], '-', ['Si', 'sp3', 0, 4, 'sg'], '-', ['C', 'sp3', 3, 1, 'sg']] \\
\hline 120 & [['C', 'sp3', 3, 1, 'sg'], '-', ['Si', 'sp3', 0, 4, 'sg'], '-', ['O', 'sp3', 0, 2, 'sg']] \\
\hline 121 & [['c', 'sp2', 0, 3, 'sg'], '-', ['S', 'sp3d3', 0, 4, 'sg'], '-', ['O', 'sp3', 1, 1, 'sg']] \\
\hline 122 & [['c', 'sp2', 0, 3, 'sg'], '-', ['S', 'sp3d3', 0, 4, 'sg'], '=', ['O', 'sp2', 0, 1, 'sg']] \\
\hline 123 & [['O', 'sp3', 1, 1, 'sg'], '-', ['S', 'sp3d3', 0, 4, 'sg'], '=', ['O', 'sp2', 0, 1, 'sg']] \\
\hline 124 & [['O', 'sp2', 0, 1, 'sg'], '=', ['S', 'sp3d3', 0, 4, 'sg'], '=', ['O', 'sp2', 0, 1, 'sg']] \\
\hline
\end{tabular}

\subsection{Hyperparameter Tuning for RFC}

The RFC model contains several hyperparameters that need to be tuned to obtain the optimal model performance. An exhaustive grid search algorithm with 5-fold cross validation (GridSearchCV in Scikitlearn) is used to obtain the best set of hyperparameters. This hyperparameter tuning is performed only for the two extreme cases, No-chem (without using fingerprints), and with Morgan fingerprints. For all the cases incorporating fingerprints, the parameters obtained via tuning for the Morgan-fp case are used. Below are the values of the hyperparameter ranges considered for GridSearchCV:

n_estimators: $[50,100,200,300]$

max_depth: [10, 20, 50,100, None]

max_features: ['sqrt', $0.25,0.5,0.75,1.0]$

min_samples_split: $[2,4,6]$

min_impurity_decrease: [0.0, 0.05, 0.1]

criterion: ['gini', 'entropy']

bootstrap: [True, False]

Table S5: Optimal values of hyperparameters obtained using GridSearchCV with 5-fold cross-validation for the No-chem and Morgan-fp case. The parameters obtained for the Morgan-fp case are used for all the descriptors as well.

\begin{tabular}{lcc}
\hline \hline Parameter & No-Chem & Morgan-fp \\
\hline bootstrap & False & False \\
ccp_alpha & 0.0 & 0.0 \\
class_weight & None & None \\
criterion & gini & gini \\
max_depth & 50 & 50 \\
\hline \hline
\end{tabular}




\begin{tabular}{lcc}
\hline \hline max_features & sqrt & sqrt \\
max_leaf_nodes & None & None \\
max_samples & None & None \\
min_impurity_decrease & 0.0 & 0.0 \\
min_impurity_split & None & None \\
min_samples_leaf & 1 & 1 \\
min_samples_split & 2 & 2 \\
min_weight_fraction_leaf & 0.0 & 0.0 \\
n_estimators & 200 & 200 \\
\hline \hline
\end{tabular}

Table S6: Model accuracies for the No-chem and Morgan-fp cases obtained using the GridSearchCV algorithm with 5-fold cross validation.

\begin{tabular}{lcc}
\hline \hline Estimator & Accuracy & 1-(Hamming loss) \\
\hline \multicolumn{1}{l}{ No-chem } & & \\
\hline 4-fold CV (training set) & $0.925 \pm 0.012$ & $0.985 \pm 0.008$ \\
Best estimator (testing set) & $0.928 \pm 0.004$ & $0.988 \pm 0.002$ \\
\hline & Morgan-fp & \\
\hline 4-fold CV (training set) & $0.919 \pm 0.010$ & $0.978 \pm 0.006$ \\
Best estimator (testing set) & $0.922 \pm 0.003$ & $0.982 \pm 0.004$ \\
\hline \hline
\end{tabular}

\section{SCFT Accuracy}

SCFT, being a coarse-grained theory, predicts universal phase behavior (order-disorder and order-order transition boundaries) as a function of $\chi_{A B} N$ and $f_{A}$. In order to compute the accuracy of SCFT, the blocks' chemistries, $T$, and $M_{n}$ for all the data points are transformed to their respective $\chi_{A B} N$ values using Eqs. (1) and (2), and with solubility parameters obtained using method described in Section 1. Then, a SCFTpredicted phase is assigned to each data point based on the location of its corresponding state point $\left(\chi_{A B} N, f_{A}\right)$ in the SCFT phase diagram. For example, if a data point has $\left(\chi_{A B} N, f_{A}\right)=(40,0.45)$, it is assigned a Lam phase, as the state point $(40,0.45)$ lies in the Lam region predicted by SCFT (Fig. 1(b) in the main manuscript). Similarly, all the data points are assigned to their respective SCFT-predicted phase according to their $\left(\chi_{A B} N, f_{A}\right)$ values, which constitute a vector of SCFT predictions. It is well-established that SCFT-predicted phase boundaries depend on the blocks conformational asymmetric $\left(b_{A} / b_{B}\right){ }^{9}$ However, generating SCFT phase diagrams for all the different values of conformational asymmetry that exist in the database is computationally very expensive process. Hence, throughout this work, the SCFT phase diagram of conformational symmetric diblock copolymers $\left(b_{A}=b_{B}\right)$ is used to compute the SCFT accuracy. For IS diblock copolymers $b_{I}=6.5 \AA$, and $b_{S}=6.7 \AA$ at $T=140{ }^{\circ} \mathrm{C},{ }^{1}$ yielding $b_{S} / b_{I}=1.031$ which is almost equal to 1 , hence, the accuracy for IS diblocks is also computed using a conformationally symmetric phase diagram. An open-source SCFT code, polymer self-consistent field (PSCF), is used to construct the SCFT phase diagram of diblock copolymers. ${ }^{10}$ Moreover, only the five classical phases: lamellar (Lam), hexagonal cylinders (Cyl), BCC sphere (Sph), gyroid (Gyr), and disordered (Dis) are considered to be the competing phases. 
Once the $\left(\chi_{A B} N, f_{A}\right)$ values of all the datapoints are known, the accuracies of SCFT prediction are computed using Eqs. (3) and (4) and the list of true (experimentally-observed) phases. There is an infinitesimal chance that the transformed $\left(\chi_{A B} N, f_{A}\right)$ values for the phase transition points will lie exactly on the respective phase boundaries predicted by SCFT. Therefore, the phase transition points will have an infinitesimal chance to be predicted correctly by SCFT. In order to correct for this numerical limitation, accuracies are also computed by discarding the phase transition data points, and this case is referred to as the single-phase case. Moreover, for the single-phase case, accuracy $(A)$ and $1-$ (Hamming loss) are not independent, and related as $A=1-5 / 2 *$ (Hamming loss). Below are the accuracies of SCFT for all the cases examined:

Table S7: Accuracies for SCFT predictions using various functional form of $\chi_{A B}$. Here, the single-phase case refers to the analysis in which only the data points that are observed as a single phase are considered, while the phase transition points (order-order and order-disorder transitions) are excluded. It can be shown that for the single-phase case, the accuracy (A) and Hamming loss $(\mathrm{H})$ are related as $H=2 / 5(1-A)$.

\begin{tabular}{lcc}
\hline \hline$\chi_{A B}$ transformation method & Test accuracy & $1-($ Hamming loss $)$ \\
\hline$\chi_{A B}=\left(\delta_{A}-\delta_{B}\right)^{2} v_{\text {ref }} / R T$ & 0.1875 & 0.6884 \\
$\chi_{A B}=\left(\delta_{A}-\delta_{B}\right)^{2} v_{\text {ref }} / R T+0.34$ & 0.5285 & 0.8234 \\
$\chi_{A B}=\left(\delta_{A}-\delta_{B}\right)^{2} v_{\text {ref }} / R T$ (Single-phase) & 0.2194 & 0.6878 \\
$\chi_{A B}=\left(\delta_{A}-\delta_{B}\right)^{2} v_{\text {ref }} / R T+0.34$ (Single-phase) & 0.6184 & 0.8474 \\
$\chi_{I S}=\left(\delta_{A}-\delta_{B}\right)^{2} v_{\text {ref }} / R T$ & PI-PS (IS) diblock copolymers & \\
$\chi_{I S}=\left(\delta_{A}-\delta_{B}\right)^{2} v_{\text {ref }} / R T+0.34$ & 0.1183 & 0.6698 \\
$\chi_{I S}=71.4 / T-0.0857$ & 0.5382 & 0.8245 \\
$\chi_{I S}=\left(\delta_{A}-\delta_{B}\right)^{2} v_{\text {ref }} / R T$ (Single-phase) & 0.5382 & 0.8245 \\
$\chi_{I S}=\left(\delta_{A}-\delta_{B}\right)^{2} v_{\text {ref }} / R T+0.34$ (Single-phase) & 0.1402 & 0.6561 \\
$\chi_{I S}=71.4 / T-0.0857$ (Single-phase) & 0.6376 & 0.8550 \\
\hline \hline
\end{tabular}

\section{RFC Model Accuracy and Feature Importance Scores}

Table S8: Numerical values of the feature importance scores obtained using RFC model for different fingerprint types, plotted in Figure 2 of the main manuscript. The score for Chemistry is calculated as the sum of scores for all the individual components in the fingerprint vector. The length of the different fingerprint vectors used are: GCT $=1$, Singlets $=22$, Doublets $=52$, Triplets $=124$, Morgan $-\mathrm{fp}=1024$, RDKit-fp $=1024$, and RDKit-desc $=109$.

\begin{tabular}{lcccc}
\hline \hline Fingerprint type & $\boldsymbol{f}_{\boldsymbol{A}}$ & $\boldsymbol{M}_{\boldsymbol{n}}(\mathbf{g} / \mathbf{m o l})$ & $\boldsymbol{T}(\mathbf{K})$ & Chemistry \\
\hline No-chem & $0.354 \pm 0.004$ & $0.281 \pm 0.003$ & $0.365 \pm 0.005$ & -- \\
GCT & $0.262 \pm 0.003$ & $0.310 \pm 0.004$ & $0.281 \pm 0.004$ & $0.147 \pm 0.003$ \\
Singlets & $0.195 \pm 0.005$ & $0.328 \pm 0.004$ & $0.249 \pm 0.006$ & $0.227 \pm 0.003$ \\
Doublets & $0.192 \pm 0.004$ & $0.336 \pm 0.004$ & $0.243 \pm 0.004$ & $0.228 \pm 0.003$ \\
Triplets & $0.190 \pm 0.005$ & $0.341 \pm 0.005$ & $0.235 \pm 0.005$ & $0.234 \pm 0.004$ \\
Morgan-fp & $0.195 \pm 0.003$ & $0.354 \pm 0.003$ & $0.221 \pm 0.003$ & $0.229 \pm 0.003$ \\
RDKit-fp & $0.196 \pm 0.004$ & $0.358 \pm 0.004$ & $0.223 \pm 0.005$ & $0.223 \pm 0.003$ \\
\hline \hline
\end{tabular}




\begin{tabular}{lllll}
\hline \hline RDKit-Desc & $0.199 \pm 0.003$ & $0.345 \pm 0.005$ & $0.224 \pm 0.003$ & $0.231 \pm 0.003$ \\
\hline \hline
\end{tabular}

Table S9: Test and Hamming accuracies obtained from the RFC that are plotted in Figure 2 of the main manuscript. These metrics are computed on a test sample which is $25 \%$ of the total data points. Moreover, the test sample is same across all the fingerprint types.

\begin{tabular}{lcc}
\hline \hline Fingerprint type & Accuracy & $\mathbf{1}-$ (Hamming loss) \\
\hline No-chem & $0.926 \pm 0.005$ & $0.980 \pm 0.001$ \\
GCT & $0.943 \pm 0.003$ & $0.985 \pm 0.001$ \\
Singlets & $0.922 \pm 0.002$ & $0.981 \pm 0.001$ \\
Doublets & $0.921 \pm 0.002$ & $0.980 \pm 0.001$ \\
Triplets & $0.921 \pm 0.002$ & $0.980 \pm 0.001$ \\
Morgan-fp & $0.921 \pm 0.002$ & $0.980 \pm 0.001$ \\
RDKit-fp & $0.920 \pm 0.003$ & $0.979 \pm 0.001$ \\
RDKit-Desc & $0.921 \pm 0.002$ & $0.980 \pm 0.001$ \\
\hline \hline
\end{tabular}

Table S10: Test and Hamming accuracies obtained from the RFC model only for the PI-PS (IS) diblock copolymers for No-chem and Morgan-fp cases. Total number of datapoints for IS diblock copolymers are 693.

\begin{tabular}{lcc}
\hline \hline Fingerprint type & Accuracy & $\mathbf{1}$-(Hamming loss) \\
\hline No-chem & $0.929 \pm 0.009$ & $0.982 \pm 0.002$ \\
\hline Morgan-fp & $0.916 \pm 0.008$ & $0.980 \pm 0.002$ \\
\hline \hline
\end{tabular}

\section{Effect of Chemically Identical Blocks}

An important feature of a model developed to predict block copolymer phase behavior is that the model should yield a disordered phase if the two blocks are of same chemical groups. This fundamental physics is typically inherent to physics-based models such as SCFT, however, for data-driven model such physics needs to built-in by supplying additional data. This section explores this possibility by appending the dataset with artificially created data points, with each datapoint having the two blocks of same chemical groups, and yielding a disordered phase. Specifically, for each chemical group A present in the dataset, a new data point is created as:

Table S11: An example of new data point (A, A, $f_{A}^{i}, T^{i}, M_{n}^{i}$ ) created in the dataset with two blocks having the same chemical group, and yielding a disordered phase $(0,0,0,0,1)$. Here A represent any one of the chemical groups present in the dataset.

\begin{tabular}{cccccccccc}
\hline \hline Block 1 & Block 2 & $\boldsymbol{f}_{\boldsymbol{A}}$ & $\boldsymbol{T}$ & $\boldsymbol{M}_{\boldsymbol{n}}$ & Lam & Gyr & Cyl & Sph & Dis \\
\hline \hline $\mathrm{A}$ & $\mathrm{A}$ & $f_{A}^{i}$ & $T^{i}$ & $M_{n}^{i}$ & 0 & 0 & 0 & 0 & 1 \\
\hline
\end{tabular}

The values of $f_{A}^{i}, T^{i}$, and $M_{n}^{i}$, are selected by making a uniformly spaced 3-dimensional grid of $n_{f_{A}} \times n_{T} \times n_{M_{n}}$ points with the maximum and minimum of grid chosen as the maximum and minimum of the respective feature in the dataset. This results in a $n_{f} \times n_{T} \times n_{M_{n}}$ new datapoints 
for each chemistry in the dataset. To minimize the influence of these artificially created datapoints on the overall model, a minimum reasonable value is chosen: $n_{f_{A}}=n_{T}=n_{M_{n}}=5$, leading to 125 additional data points for each chemical group with each representing the disordered phase. The model is then trained with a regular procedure of 75:25 train-test split, but with a new dataset consisting of 13,411 datapoints.
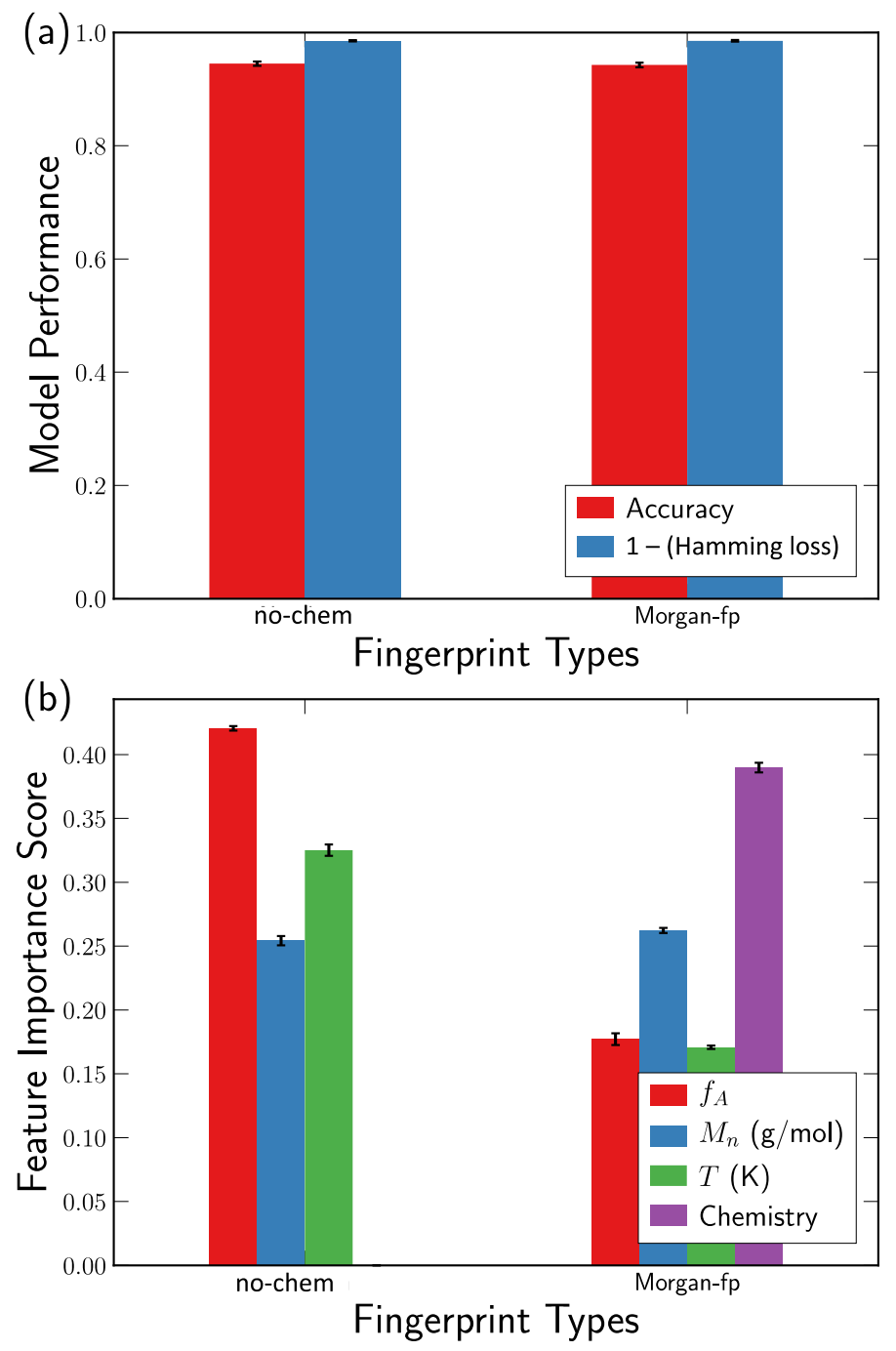

Figure S3: RFC modeling of a data set which is appended by additional datapoints representing disordered phase when the two block chemistries are same. (a) Performance of the RFC model, characterized by accuracy and 1 - (Hamming loss), and (b) Ranking of different features as indicated by the respective importance score, with the total score being equal to unity. These metrics are computed on 25\% (test set) of the dataset consisting of 13,411 data points, and with a 4-fold cross-validation procedure.

Figure S3 shows that the model trained with the new dataset still maintains an overall accuracy of approximately $90 \%$, however, the feature ranking is changed compared to the model developed using the original dataset. Since the new dataset contains 125 additional datapoints for each chemistry, the influence of chemistry on developing a model is increased significantly as demonstrated by chemistry feature importance score in Figure S3(b). 
To test the model strictly for the fundamental physics of yielding a disordered phase with the two blocks being chemically identical, 100 new data points are created for each chemistry similar to

shown in Table $\mathrm{S} 1$, but with $f_{A}^{i}, T^{i}$, and $M_{n}^{i}$ chosen from a uniform distribution between the maximum and minimum of respective feature. The model trained with Morgan-fp yields $100 \%$ accuracy of identifying a disordered phase on the above new test set containing exclusively data points with two blocks being same. It is remarkable that even with 125 additional datapoints for each chemistry in the training set, the model is able to capture the essential physics of selfassembly. It is worth mentioning that the model trained without fingerprints (No-chem case) yields almost half the accuracy $(51 \%)$ on this new dataset, highlighting the importance of chemical fingerprints in developing a data-driven model for block copolymer self-assembly.

\section{Effects on Fingerprinting Methods on Model Performance}

The unexpected near-equivalent performance of the model for all fingerprinting methods attempted behavior is likely due to three reasons. First, it is possible that the cumulative effect of chemistry can be captured by several different combinations of descriptors, provided the total number of descriptors describing a single chemistry is sufficiently large. This is prominent in the performances of Triplets, Morgan-fp, RDKit-fp, and RDKit-Desc which differ by less than $0.2 \%$ (Table S9), with each of these cases containing at least 100 descriptors per block. Second, the data set may not be sufficiently chemically diverse; as a result, the difference in chemical incompatibility $\chi_{A B}=\alpha / T+\beta$ is dominated by the variation in temperature, and less affected by block chemistries. Lastly, the data is heavily structured in $\left(T, f_{\mathrm{A}}, M_{n}\right)$ as depicted in Figure 1. One prominent structure is the streaks at constant $f_{A}$ with closely spaced temperature intervals that arise from temperature sweeps in scattering and rheological measurements. While creating a decision tree, this structure allows the RFC model to lean on a few initial crucial splits based on $f_{A}$, followed by splits based on $T$, to make accurate predictions without ever considering blocks chemistries. Accordingly, the structure in $\left(T, f_{\mathrm{A}}, M_{n}\right)$ can have a pronounced influence on the overall classification process.

In many experimental designs, a significant amount of data is collected using rheology and SAXS measurements which are recorded with reasonably high frequency $\left(\Delta T=5-10^{\circ} \mathrm{C}\right)$. This creates an extremely high density of points along the $T$ axis at constant values of $f_{A}$, as depicted by Figure 1 (a) of the main manuscript. In order to reduce such a high density and an a priori biased towards temperature, data points are removed by randomly discarding $50 \%$ and $90 \%$ of the data points along the $T$ axis at constant $f_{A}$. These datasets are names as DS_red50 and DS_red90, respectively. Moreover, this reduction of data is performed only for those $f_{A}$ values for which the number of data points along the $T$ axis is greater than 25 . Below are the results (accuracies and feature importance scores) of the RFC model using the resulting smaller datasets.

For each of the two reduced datasets, two different analyses are performed. In the first case, the RFC model is trained with $75 \%$ of the smaller dataset and tested on the remaining $25 \%$ dataset. It is observed that the accuracies drop for both the smaller data sets, with the DS_red90 demonstrating only 75\% accuracy (Table S13). In the second analysis, the whole smaller dataset is used for training the model, and the accuracy is computed on the larger data set that comprises of all the datapoints in the original dataset (9536) minus the datapoints in the smaller dataset. In this case also, the test accuracies decrease for both the smaller datasets, approximately $82 \%$ for DS_red50 and 75\% for DS_red90 (Table S14). To further understand this decrease in accuracy, the remaining dataset is further discretized into two categories: a sparse dataset that contains points which do not form temperature sweeps, and a non-sparse dataset which contains points that are 
randomly removed from temperature sweeps. The accuracy for the sparse dataset is significantly lower, approximately 68\% for DS_red50 and 40\% for DS_red90, while the accuracy for the non-sparse dataset remains high, 93\% for DS_red50 and 82\% for DS_red90. This high accuracy of non-sparse remaining dataset is not surprising considering that these datapoints belong to the high-density temperature sweeps, and hence are expected to be predicted correctly by a model that is trained on the low-density datapoints of the same temperature sweeps. The lower accuracy on the sparse portion of the data demonstrates the common behavior of machine-learning models, i.e., predicting the high-density data correctly at the expense of misclassifying low-density data. Nevertheless, this whole analysis demonstrates that only a few rheology and scattering temperature measurements reported at moderate to small intervals are sufficient to develop a reasonably accurate model.

Table S12: Test and Hamming accuracies obtained from the RFC model on reduced smaller datasets. For each of the reduce datasets, the RFC model is trained on $75 \%$ of the dataset and accuracy is computed on the remaining $25 \%$ of the dataset.

\begin{tabular}{|c|c|c|c|c|c|c|}
\hline \multirow[t]{2}{*}{ Fingerprint type } & \multicolumn{2}{|c|}{ Model Performance } & \multicolumn{4}{|c|}{ Feature Importance Scores } \\
\hline & Accuracy & 1 - (Hamming loss $)$ & $f_{A}$ & $\mathbf{T}(\mathbf{K})$ & $M_{n}(\mathrm{~g} / \mathrm{mol})$ & Chemistry \\
\hline \multicolumn{7}{|c|}{ DS_red50: 5323 data points } \\
\hline No-chem & $0.876 \pm 0.008$ & $0.968 \pm 0.002$ & $\begin{array}{l}0.341 \\
\pm 0.005\end{array}$ & $\begin{array}{l}0.369 \\
\pm 0.005\end{array}$ & $\begin{array}{l}0.289 \\
\pm 0.004\end{array}$ & -- \\
\hline Morgan-fp & $0.874 \pm 0.008$ & $0.967 \pm 0.002$ & $\begin{array}{l}0.200 \\
\pm 0.003\end{array}$ & $\begin{array}{l}0.234 \\
\pm 0.003\end{array}$ & $\begin{array}{l}0.343 \\
\pm 0.003\end{array}$ & $\begin{array}{l}0.221 \\
\pm 0.005\end{array}$ \\
\hline \multicolumn{7}{|c|}{ DS_red90: 1944 data points } \\
\hline No-chem & $0.749 \pm 0.006$ & $0.930 \pm 0.008$ & $\begin{array}{l}0.330 \\
\pm 0.006\end{array}$ & $\begin{array}{l}0.374 \\
\pm 0.008\end{array}$ & $\begin{array}{l}0.295 \\
\pm 0.004\end{array}$ & -- \\
\hline Morgan-fp & $0.743 \pm 0.004$ & $0.925 \pm 0.007$ & $\begin{array}{l}0.209 \\
\pm 0.006\end{array}$ & $\begin{array}{l}0.260 \\
\pm 0.004\end{array}$ & $\begin{array}{l}0.289 \\
\pm 0.008\end{array}$ & $\begin{array}{l}0.241 \\
\pm 0.009\end{array}$ \\
\hline
\end{tabular}

Table S13: Test and Hamming accuracies obtained from the RFC model on reduced smaller datasets. For each of the reduce datasets, the RFC model is trained on the complete reduced dataset, and the accuracy is computed on the dataset that comprises of all the datapoints in the original dataset (9536) excluding the datapoints that are in the reduced dataset. This analysis does not require a test-train split, and hence it does not have standard deviation associated with the accuracies and scores.

\begin{tabular}{lcccccc}
\hline \hline Fingerprint type & \multicolumn{1}{c}{ Model Performance } & \multicolumn{3}{c}{ Feature Importance Scores } \\
\hline \hline & Accuracy & 1 - (Hamming loss) & $\boldsymbol{f}_{\boldsymbol{A}}$ & $\boldsymbol{T}(\mathbf{K})$ & $\boldsymbol{M}_{\boldsymbol{n}}(\mathrm{g} / \mathbf{m o l})$ & Chemistry \\
\hline \hline No-chem & $0.812 \pm 0.008$ & $0.940 \pm 0.002$ & 0.354 & 0.347 & 0.299 & -- \\
Morgan-fp & $0.829 \pm 0.009$ & $0.943 \pm 0.002$ & 0.169 & 0.194 & 0.390 & 0.248 \\
\hline \multicolumn{7}{c}{ DS_red50: 5323 data points } \\
\hline No-chem & 0.755 & DS_red90: 1944 data points & & \\
Morgan-fp & 0.764 & 0.929 & 0.320 & 0.344 & 0.336 & -- \\
\hline \hline
\end{tabular}




\section{Effect of Chemical Diversity}

In general, the performance as well as reliability of a machine-learning model depends critically on the number and types of chemical groups exist in the dataset. In order to explore the effect of chemical diversity on the model performance, a smaller dataset is constructed by removing the data points corresponding to the following 9 chemical groups (selected randomly) in the original dataset:

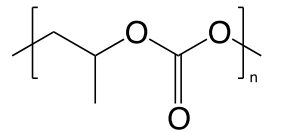

Polypropylene carbonate

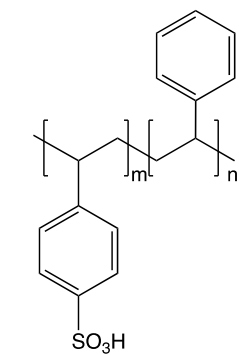

Poly(styrene sulfonate)-rpolystyrene

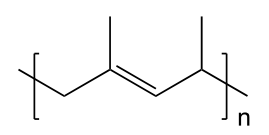

Poly(2-methyl pentadiene) (P2MP)

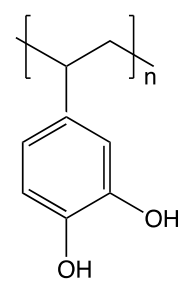

Poly(dihydroxy styrene)

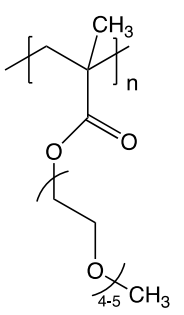

Poly(oligoethylene glycol methyl ether methacrylate) (POEGMA)

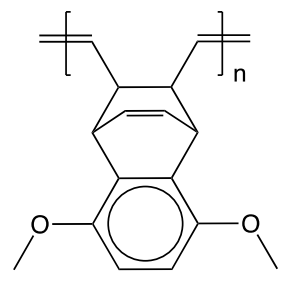

Poly-pdmb-precursor ${ }^{4}$

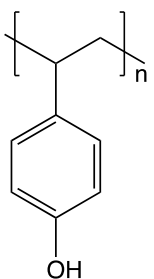

Poly(4-hydroxy styrene)
Polyethylene-rpolyethyl ethylene

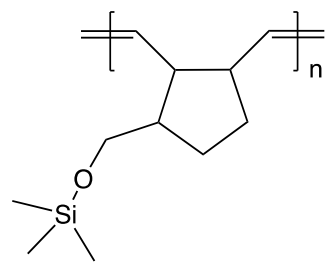

Poly[((trimethylsiloxy)methyl)norbomene] ${ }^{4}$

Figure S4: Chemical groups that are removed to create a less-diverse dataset. Here, only one repeat unit generated using a SMILES representation via RDKit is depicted, however, for developing the RFC model, oligomers of 8 repeat units with last and first atoms joined, are used.

The reduced dataset, comprising of 9440 points (original dataset contains 9536 points), is then used as input to the whole procedure described above, involving a 75-25 test-train split in stratified manner, followed by the training of RFC model. Figure S2 shows the accuracies and feature importance scores obtained using the test set of the reduced dataset. While the model performance remains almost the same, the ranking of features governing the phase behavior is significantly different compared to those obtained using a complete dataset (Figure 2 of the main manuscript). For the cases with chemical fingerprints, the less-diverse dataset yields temperature to be the most dominant feature while the original dataset yields molar mass as the most important feature. Similarly, the No-chem and GCT cases also yields different ranking of features for the less-diverse dataset compare to the original dataset. 


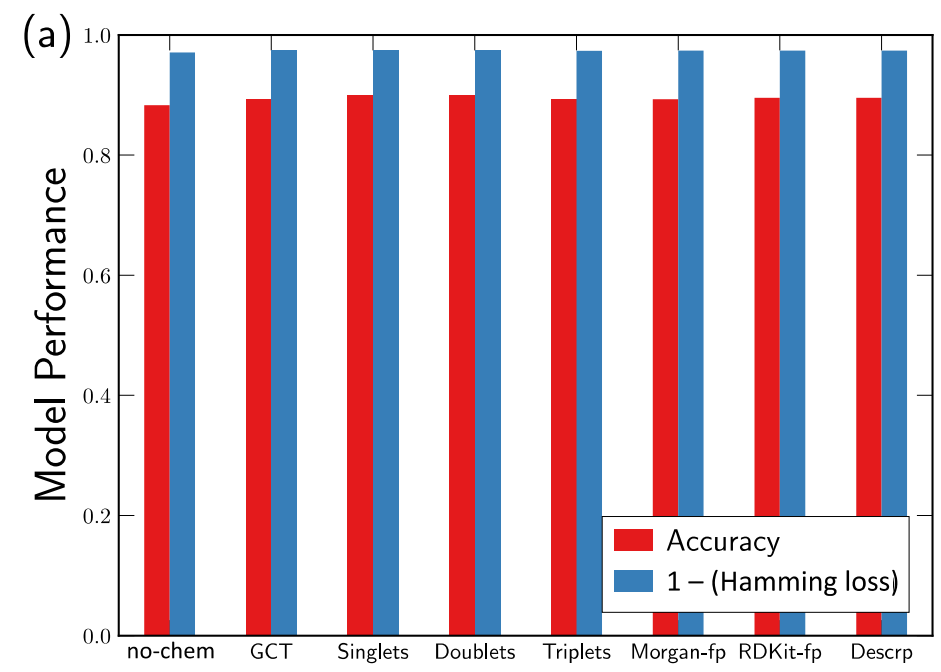

Fingerprint Types

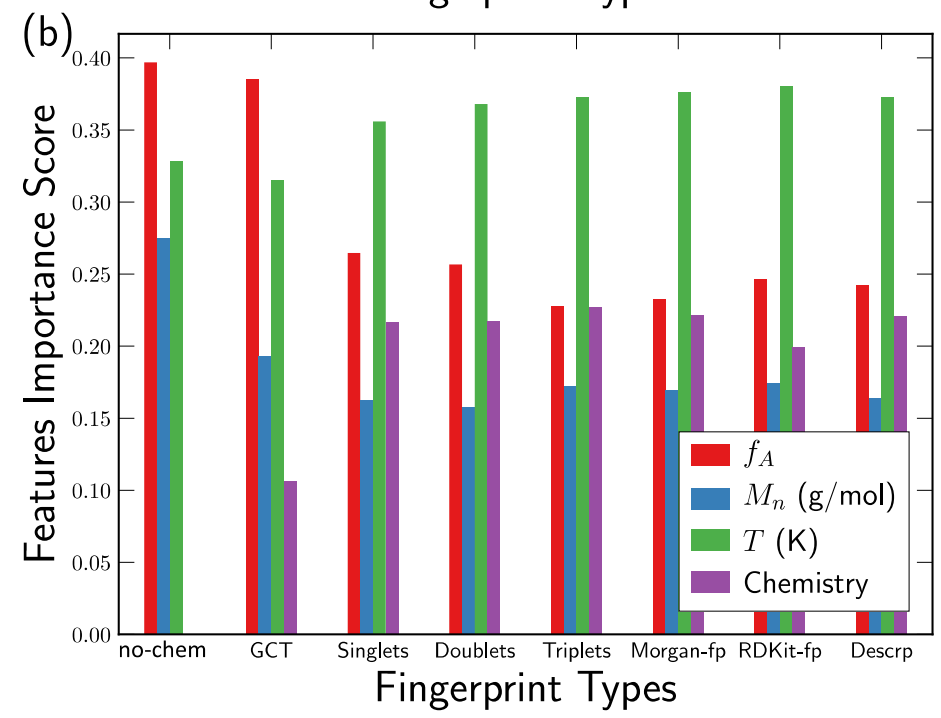

Figure S5: RFC modeling of a data set with reduced chemical diversity. (a) Performance of the RFC model, characterized by accuracy and 1 - (Hamming loss), for various types of fingerprinting methods. (b) Ranking of different features as indicated by the respective importance score, with the total score being equal to unity. These metrics are computed on $25 \%$ (test set) of the reduced smaller dataset, and a 4-fold crossvalidation procedure. 


\section{Extrapolation to Various Chemistries}

A particularly useful feature of data-driven model would be that it extrapolates to various other chemistries so that it can be used to discover new diblock copolymers for desired ordered structure. To test such an extrapolation capability of the model, the dataset is divided into two sets: a small set containing only a few selected diblocks, and a large set containing all the remaining diblocks. The model is trained on the smaller dataset and it is tested on the large dataset containing many different chemical groups. This procedure is performed for three different small sets: $\{\mathrm{PI}-\mathrm{PS}\},\{\mathrm{PI}-$ PS, PEE-PEO, PEP-PDMS \}, \{PI-PS, PEE-PEO, PEP-PDMS, PI-PLA, P2VP-PDMS \}. Table S11 demonstrates that the model trained on the smaller dataset yields significantly lower accuracy, approximately $26 \%$ when tested on the large dataset containing remaining diblocks.

In addition to training the model with a few selected diblocks, the analysis was also performed by training the model with large set of chemistries and testing on the remaining small set of chemistries. Specifically, out of all M chemistries that exist in the dataset, the model is trained on M-1 chemistries, and tested on the remaining one chemistry. This procedure is repeated for all M chemistries, and the results are averaged over these M model predictions. Table S12 shows that the model trained by removing only one chemistry out of 31 chemistries, still do not perform well in predicting the phase behavior of the remaining chemistry, as evident by accuracy of approximately $22 \%$ accuracy. A detailed investigation of this analysis demonstrates that several chemistries have a very small number of datapoints (15-20), and many of those data points are coexisting phases which are difficult to be predicted correctly. As a result, out of these $\mathrm{M}$ tests, several tests yield accuracy of 0 but $1-$ (Hamming loss) of greater than $50 \%$. Another consequence of this disproportion in number of points for a given chemistry is that the variance in accuracy is very high for this procedure, as depicted by Figure S5, and listed in Tables S12 and S13. Overall, the analyses presented both the tests performed in this section highlight that with the present chemical diversity and distribution of datapoints for each chemistry, the model is not very useful is discovering new structures, however, with the addition of more diverse datapoints for each chemistry, the extrapolation power of the model can be increased further.

Table S14: Accuracy and 1 - (Hamming loss) obtained from the RFC model that is trained on selected diblock copolymers, and tested on the remaining diblock copolymers in the dataset. Captions in the table below indicate the diblocks selected for training the model, and the number of data points associated with the chosen set of diblocks.

\begin{tabular}{lcc}
\hline \hline Fingerprint Type & Accuracy & $\mathbf{1}-$ (Hamming loss) \\
\hline \hline \multicolumn{3}{c}{ Training diblocks: $\{$ PI-PS $\}$ (1386 data points) } \\
\hline Morgan-fp & $0.276 \pm 0.004$ & $0.738 \pm 0.002$ \\
RDKit-fp & $0.282 \pm 0.008$ & $0.743 \pm 0.002$ \\
\hline \hline \multicolumn{4}{c}{ Morgan-fp } & Training diblocks: & PI-PS, PEE-PEO, PEP-PDMS $\}$ (2650 data points) \\
RDKit-fp & $0.261 \pm 0.006$ & $0.742 \pm 0.008$ \\
\hline \hline & $0.264 \pm 0.004$ & $0.744 \pm 0.007$ \\
\hline Morgan-fp & Training diblocks: $\{$ PI-PS, PEE-PEO, PEP-PDMS, PI-PLA, P2VP-PDMS $\}(3606$ data points) \\
RDKit-fp & $0.234 \pm 0.008$ & $0.745 \pm 0.008$ \\
\hline \hline
\end{tabular}


Table S15: Accuracy and 1 - (Hamming loss) obtained from the RFC model that is trained on M-1 chemistries and tested on the remaining 1 chemistry, where $\mathrm{M}$ is the total number of chemistries in the dataset. The results show the average of $\mathrm{M}$ tests performed.

\begin{tabular}{lcc}
\hline \hline Fingerprint Type & Accuracy & $\mathbf{1 - ( H a m m i n g ~ l o s s )}$ \\
\hline \hline & Training diblocks: $\{$ PI-PS $\}$ (1386 data points) & \\
\hline Morgan-fp & $0.265 \pm 0.200$ & $0.796 \pm 0.066$ \\
RDKit-fp & $0.230 \pm 0.246$ & $0.778 \pm 0.077$ \\
\hline \hline
\end{tabular}
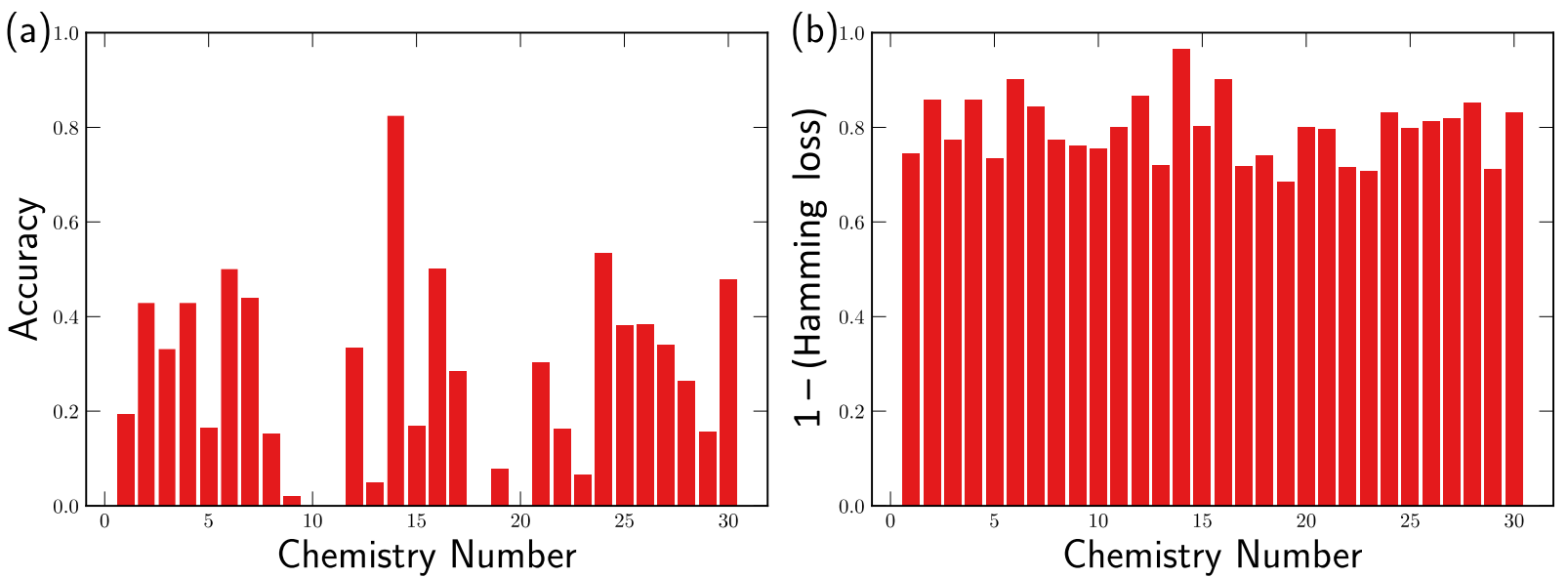

Figure S6: (a) Accuracy, and (b) 1 - (Hamming loss) obtained from the RFC model that is trained on M-1 chemistries and tested on the remaining 1 chemistry, where $M(=30)$ is the total number of chemistries in the dataset. Chemistries corresponding to the chemical number are listed in Table S16.

Table S16: SMILES string, number for datapoints, and numerical values of accuracies for different chemistries for the test presented in Figure S6. The chemistry number listed in first column corresponds to the chemistry number plotted in Figure S6.

\begin{tabular}{|c|c|c|c|c|c|}
\hline $\begin{array}{l}\text { Chemistry } \\
\text { Number }\end{array}$ & Polymer & BigSMILES & $\begin{array}{l}\text { Number of } \\
\text { datapoints }\end{array}$ & Accuracy & $1-$ (Hamming loss) \\
\hline 1 & Poly(lactic acid) & $\{[][<] \mathrm{C}(\mathrm{C}) \mathrm{C}(=\mathrm{O}) \mathrm{O}[>][]\}$ & 882 & 0.194 & 0.744 \\
\hline 2 & $\begin{array}{l}\text { Poly[((trimethylsiloxy)- } \\
\text { methyl)norbornene }]^{4}\end{array}$ & $\begin{array}{l}\{[][\$]=\mathrm{CC}(\mathrm{C} 1) \mathrm{CC}(\mathrm{CO}[\mathrm{Si}] \\
(\mathrm{C})(\mathrm{C}) \mathrm{C}) \mathrm{C} 1 \mathrm{C}=[\$][]\}\end{array}$ & 14 & 0.429 & 0.857 \\
\hline 3 & Poly(oxybutylene) & $\{[][<] \mathrm{C}(\mathrm{CC}) \mathrm{CO}[>][]\}$ & 148 & 0.331 & 0.773 \\
\hline 4 & Poly-pdmb-precursor ${ }^{4}$ & $\begin{array}{l}\{[][\$]=\mathrm{CC} 1 \mathrm{C}(\mathrm{C} 3 / \mathrm{C}=\mathrm{ClC} 1 \\
\mathrm{c} 2 \mathrm{c}(\mathrm{OC}) \operatorname{ccc}(\mathrm{OC}) \mathrm{c} 23) \mathrm{C}=[\$ \\
][]\}\end{array}$ & 14 & 0.429 & 0.857 \\
\hline 5 & Polyethylene & $\begin{array}{l}\{[][\$] \mathrm{CCCC}[\$],[\$] \mathrm{CC}(\mathrm{CC} \\
)[\$][]\}\end{array}$ & 1814 & 0.164 & 0.735 \\
\hline 6 & Poly(tert-butyl acrylate) & $\begin{array}{l}\{[][\$] \mathrm{CC}(\mathrm{C}(=\mathrm{O}) \mathrm{OC}(\mathrm{C})(\mathrm{C}) \\
\mathrm{C})[\$][]\}\end{array}$ & 2 & 0.500 & 0.900 \\
\hline 7 & $\begin{array}{l}\text { Poly(methyl } \\
\text { methacrylate) }\end{array}$ & $\begin{array}{l}\{[][\$] C C(C)(C(=O) O C)[\$ \\
][]\}\end{array}$ & 82 & 0.439 & 0.844 \\
\hline
\end{tabular}




\begin{tabular}{|c|c|c|c|c|c|}
\hline 8 & $\begin{array}{l}\text { Poly(ethyl hexyl } \\
\text { methacrylate) }\end{array}$ & $\begin{array}{l}\{[][\$] C C(C)(C(=O) O C C( \\
\mathrm{CC}) \mathrm{CCCC})[\$][]\}\end{array}$ & 46 & 0.152 & 0.774 \\
\hline 9 & Poly(butyl methacrylate) & $\begin{array}{l}\{[][\$] C C(C)(C(=O) O C C C \\
C)[\$][]\}\end{array}$ & 50 & 0.020 & 0.760 \\
\hline 10 & POEGMA $^{a}$ & $\begin{array}{l}\{[][\$] C C(C)(C(=O)\{[>][< \\
] \mathrm{OCC}[>][<]\} O C)[\$][]\}\end{array}$ & 70 & 0.000 & 0.754 \\
\hline 11 & $\begin{array}{l}\text { Poly(alphamethyl } \\
\text { styrene) }\end{array}$ & $\begin{array}{l}\{[][\$] C C(C)(\operatorname{clccccc} 1)[\$] \\
[]\}\end{array}$ & 2 & 0.000 & 0.800 \\
\hline 12 & $\mathrm{P} 2 \mathrm{MP}$ & $\begin{array}{l}\{[][\$] \mathrm{ClC}(\mathrm{C})=\mathrm{C} / \mathrm{C}(\mathrm{C})[\$],[ \\
\$] \mathrm{ClC}(\mathrm{C})=\mathrm{ClC}(\mathrm{C})[\$],[\$] \mathrm{C} \\
\mathrm{C}(\mathrm{C})(\mathrm{C}=\mathrm{CC})[\$],[\$] \mathrm{C}(\mathrm{C}) \mathrm{C} \\
(\mathrm{C}(\mathrm{C})=\mathrm{C})[\$][]\}\end{array}$ & 6 & 0.333 & 0.867 \\
\hline 13 & Poly(oxypropylene) & $\{[][<] \mathrm{CC}(\mathrm{C}) \mathrm{O}[>][]\}$ & 20 & 0.050 & 0.720 \\
\hline 14 & $\begin{array}{l}\text { Poly(propylene } \\
\text { carbonate) }\end{array}$ & $\begin{array}{l}\{[][<] \mathrm{CC}(\mathrm{C}) \mathrm{OC}(=\mathrm{O}) \mathrm{O}[>][ \\
]\}\end{array}$ & 108 & 0.824 & 0.965 \\
\hline 15 & Poly(vinyl cyclohexane) & $\begin{array}{l}\{[][\$] \mathrm{CC}(\mathrm{C} 1 \mathrm{CCCCC} 1)[\$] \\
[]\}\end{array}$ & 1088 & 0.169 & 0.802 \\
\hline 16 & Poly(1,2-butadiene) & $\{[][\$] C C(C=C)[\$][]\}$ & 4 & 0.500 & 0.900 \\
\hline 17 & Poly(ethyl ethylene) & $\{[][\$] C C(C C)[\$][]\}$ & 2686 & 0.285 & 0.718 \\
\hline 18 & $\begin{array}{l}\text { Poly }(2,5 \text {-bis[(4- } \\
\text { butoxyphenyl) } \\
\text { oxycarbonyl] styrene) } \\
\text { (PBPCS) }\end{array}$ & $\begin{array}{l}\{[][\$] C C(\operatorname{clcc}(\mathrm{C}(=\mathrm{O}) \mathrm{Oc} 2 \\
\operatorname{ccc}(\mathrm{OCCCC}) \operatorname{cc} 2) \operatorname{ccc} 1(\mathrm{C}( \\
=\mathrm{O}) \operatorname{Oc} 3 \operatorname{ccc}(\mathrm{OCCCC}) \mathrm{cc} 3)) \\
[\$][]\}\end{array}$ & 20 & 0.000 & 0.740 \\
\hline 19 & Poly(dihydroxy styrene) & $\begin{array}{l}\{[][\$] \mathrm{CC}(\mathrm{c} 1 \mathrm{ccc}(\mathrm{O}) \mathrm{c}(\mathrm{O}) \mathrm{c} 1) \\
[\$][]\}\end{array}$ & 26 & 0.077 & 0.685 \\
\hline 20 & Poly(4-hydroxy styrene) & $\begin{array}{l}\{[][\$] C C(\operatorname{clccc}(\mathrm{O}) \operatorname{cc} 1)[\$] \\
[]\}\end{array}$ & 2 & 0.000 & 0.800 \\
\hline 21 & Poly(styrene) & $\{[][\$] C C(\operatorname{c} 1 \operatorname{ccccc} 1)[\$][]\}$ & 2362 & 0.303 & 0.796 \\
\hline 22 & Poly(styrene sulfonate) & $\begin{array}{l}\{[][\$] C C(\operatorname{cocccc} 1)[\$],[\$] \\
\operatorname{CC}(\operatorname{c} 1 \operatorname{ccc}(\mathrm{S}(=\mathrm{O})(=\mathrm{O}) \mathrm{O}) \operatorname{cc} \\
1)[\$][]\}\end{array}$ & 62 & 0.161 & 0.716 \\
\hline 23 & P4VP & $\{[][\$] C C(\mathrm{c} 1 \mathrm{ccncc} 1)[\$][]\}$ & 340 & 0.065 & 0.707 \\
\hline 24 & $\mathrm{P} 2 \mathrm{VP}$ & $\{[][\$] \mathrm{CC}(\mathrm{c} 1 \mathrm{ncccc} 1)[\$][]\}$ & 880 & 0.534 & 0.831 \\
\hline 25 & Poly(isoprene) & $\begin{array}{l}\{[][\$] C \backslash C=C(C) / C[\$],[\$] C \\
\mid C=C(C) \backslash C[\$],[\$] C C(C(C) \\
=C)[\$],[\$] C C(C)(C=C)[\$] \\
[]\}\end{array}$ & 3084 & 0.382 & 0.797 \\
\hline 26 & Poly(1,4-butadiene) & $\begin{array}{l}\{[][\$] \mathrm{ClC}=\mathrm{C} / \mathrm{C}[\$],[\$] \mathrm{ClC} \\
=\mathrm{ClC}[\$],[\$] \mathrm{CC}(\mathrm{C}=\mathrm{C})[\$][] \\
\}\end{array}$ & 68 & 0.382 & 0.812 \\
\hline 27 & $\begin{array}{l}\text { PEP-or-poly(methyl } \\
\text { butylene) }\end{array}$ & $\begin{array}{l}\{[][\$] C C C(C) C[\$],[\$] C C( \\
C(C) C)[\$],[\$] C C(C)(C C)[ \\
\$][]\}\end{array}$ & 1702 & 0.340 & 0.818 \\
\hline 28 & $\begin{array}{l}\text { Polyethylene-co- } \\
\text { poly(ethyl ethylene) }\end{array}$ & $\begin{array}{l}\{[][\$] C C C C[\$],[\$] C C(C C \\
)[\$][]\}\end{array}$ & 296 & 0.264 & 0.853 \\
\hline 29 & $\begin{array}{l}\text { Poly(ethylene glycol) or } \\
\text { Poly(ethylene oxide) }\end{array}$ & $\{[][<] \mathrm{CCO}[>][]\}$ & 1920 & 0.156 & 0.710 \\
\hline 30 & Poly(dimethyl siloxane) & $\{[][<][\mathrm{Si}](\mathrm{C})(\mathrm{C}) \mathrm{O}[>][]\}$ & 1274 & 0.479 & 0.832 \\
\hline
\end{tabular}

${ }^{a}$ The number of repeat units of the oligo(ethylene glycol) side chain of POEGMA for the machine learning model was assumed to be 1 . 


\section{Sensitivity of SCFT Accuracy towards Experimental Uncertainty}

As discussed in the main manuscript, the RFC model is observed to be sensitive to the variation in experimental measurements. Similarly, the SCFT-predictions are also observed to be sensitive towards experimental uncertainty, albeit to a lesser extent compared to RFC model. Figure S6 shows plots the SCFT accuracy for variation in $f_{A}$ and $M_{n}$.
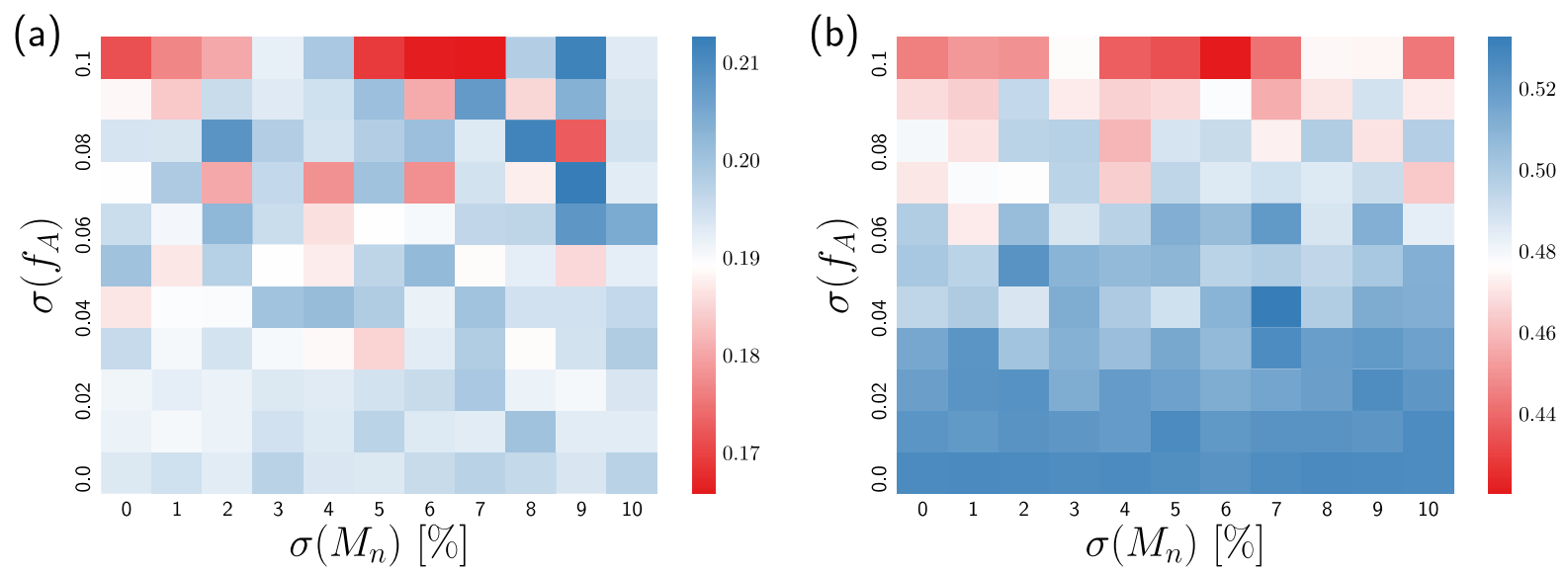

Figure S7: Heatmap for SCFT-prediction accuracy as a function of variation in $f_{A}$ and $M_{n}$ with $\Delta T=1 \mathrm{~K}$. Here, $\chi_{A B}$ is calculated using (a) Eq. (1), and (b) Eq. (2), with the solubility parameters obtained by group contribution theory as described in Section 1.

\section{References}

(1) Hiemenz, P. C.; Lodge, T. P. Polymer Chemistry; CRC press, 2007.

(2) Van Krevelen, D. W.; Te Nijenhuis, K. Properties of Polymers: Their Correlation with Chemical Structure; Their Numerical Estimation and Prediction from Additive Group Contributions; Elsevier, 2009.

(3) Bicerano, J. Prediction of Polymer Properties; cRc Press, 2002.

(4) Saunders, R. S.; Cohen, R. E.; Schrock, R. R. Synthesis and Characterization of Diblock Copolymer Films Containing Self-Assembled Polyacetylene Structures. Macromolecules 1991, 24 (20), 5599-5605.

(5) Kim, C.; Chandrasekaran, A.; Huan, T. D.; Das, D.; Ramprasad, R. Polymer Genome: A Data-Powered Polymer Informatics Platform for Property Predictions. J. Phys. Chem. C 2018, 122 (31), 17575-17585.

(6) Rogers, D.; Hahn, M. Extended-Connectivity Fingerprints. J. Chem. Inf. Model. 2010, 50 (5), 742-754.

(7) RDKit: Open-Source Cheminformatics; Http://Www.Rdkit.Org. 
(8) Sivaraman, G.; Jackson, N.; Sanchez-Lengeling, B.; Vasquez-Mayagoitia, A.; AspuruGuzik, A.; Vishwanath, V.; de Pablo, J. A Machine Learning Workflow for Molecular Analysis: Application to Melting Points. Mach. Learn. Sci. Technol. 2020.

(9) Matsen, M.; Bates, F. S. Conformationally Asymmetric Block Copolymers. J. Polym. Sci. Part B Polym. Phys. 1997, 35 (6), 945-952.

(10) Arora, A.; Qin, J.; Morse, D. C.; Delaney, K. T.; Fredrickson, G. H.; Bates, F. S.; Dorfman, K. D. Broadly Accessible Self-Consistent Field Theory for Block Polymer Materials Discovery. Macromolecules 2016, 49 (13), 4675-4690. 